\title{
Relaxation of a kinetic hole due to carrier-carrier scattering in multisubband single- quantum-well semiconductors
}

Dery, H.; Tromborg, Bjarne; Eisenstein, G.

Published in:

Physical Review B Condensed Matter

Link to article, DOI:

10.1103/PhysRevB.67.245308

Publication date:

2003

Document Version

Publisher's PDF, also known as Version of record

Link back to DTU Orbit

Citation (APA):

Dery, H., Tromborg, B., \& Eisenstein, G. (2003). Relaxation of a kinetic hole due to carrier-carrier scattering in multisubband single-quantum-well semiconductors. Physical Review B Condensed Matter, 67(24), 245308. https://doi.org/10.1103/PhysRevB.67.245308

\section{General rights}

Copyright and moral rights for the publications made accessible in the public portal are retained by the authors and/or other copyright owners and it is a condition of accessing publications that users recognise and abide by the legal requirements associated with these rights.

- Users may download and print one copy of any publication from the public portal for the purpose of private study or research.

- You may not further distribute the material or use it for any profit-making activity or commercial gain

- You may freely distribute the URL identifying the publication in the public portal 


\title{
Relaxation of a kinetic hole due to carrier-carrier scattering in multisubband single-quantum-well semiconductors
}

\author{
H. Dery, ${ }^{1, *}$ B. Tromborg, ${ }^{2}$ and G. Eisenstein ${ }^{1}$ \\ ${ }^{1}$ Department of Electrical Engineering, Technion-Israel Institute of Technology, Haifa 32000, Israel \\ ${ }^{2}$ Research Center COM, Technical University of Denmark, Building 345V, DK-2800 Lyngby, Denmark \\ (Received 28 November 2002; revised manuscript received 31 March 2003; published 12 June 2003)
}

\begin{abstract}
We describe a theoretical model for carrier-carrier scattering in an inverted semiconductor quantum well structure using a multisubband diagram. The model includes all possible nonvanishing interaction terms within the static screening approximation, and it enables one to calculate accurately the temporal evolution of the carrier densities and the gain following a perturbation by a short optical pulse. We present a theoretical formalism and detailed numerical calculations. The addition of more than one subband in each band as well as the use of all exchange terms yields several results. First, the degree of gain saturation is reduced while, at the same time, the recovery is faster as scattering events among different subbands take place. Also, carrier transfer between subbands is observed which modifies the overall carrier dynamics.
\end{abstract}

DOI: 10.1103/PhysRevB.67.245308

PACS number(s): 73.63.Hs, 85.35.Be, 42.55.Px

\section{INTRODUCTION}

The ultrafast carrier dynamics in inverted semiconductors has long been a research topic with implications in applied as well as basic semiconductor physics. Carrier dynamics is commonly investigated by assuming an inverted semiconductor quantum structure gain medium driven at high carrier densities (typical of lasers and optical amplifiers) which is perturbed by a short optical pulse which causes a spectral hole. Dynamical details are studied then by calculating the evolution of this kinetic hole. Two main theoretical approaches applicable far from equilibrium are commonly used. The first is the nonequilibrium Green's function theory using for example the time-loop $S$-matrix method ${ }^{1-4}$ and the second is the density matrix theory. ${ }^{5-8}$

This paper extends ultrafast carrier dynamics models which address the sub-1-ps time scale where carrier-carrier (c-c) scattering is the dominant mechanism. We analyze a separate confinement heterostructure $(\mathrm{SCH})$ quantum well with multiple parabolic subbands in each band using the density matrix formalism while considering all possible nonvanishing Coulomb scattering events within the multiplesubband system. A schematic description of some of the scattering possibilities including electron-electron, hole-hole, and electron-hole scattering is shown in Fig. 1.

The new general coupled equation model is accompanied by extensive numerical calculations which shed light on issues not previously observed. We demonstrate that the kinetic hole recovery is measurably faster in the multisubband case when compared to the conventional single-subband formalism. Moreover, the degree of phase filling factor saturation (which is directly related to gain saturation) is reduced in the multisubband case, as is the maximum absolute value of the polarization. Both these effects stem from Coulomb $\mathrm{c}-\mathrm{c}$ scattering events involving the second subband. The calculation also yield the time evolution of the second subband distribution which clearly shows its contribution to the overall recovery. The results obtained from the calculations are important in two ways. The addition of a second subband clearly leads to a more accurate description of this widely researched problem and, at the same time, the moderation of the role played by spectral hole burning has an important practical impact on the gain nonlinearity of quantum well (QW) lasers and amplifiers and hence on their modulation and switching capabilities.

Screening in a multisubband system is difficult to treat, since the random phase approximation (RPA) results in a dielectric matrix which must be inverted to calculate the screened potential. ${ }^{9}$ Rather than following this method, we have used the following procedure. For each specific set of subbands, we introduced the Yukawa potential (long wavelength static limit) with the inverse screening length corresponding to a two-dimensional (2D) gas with an equivalent density. This procedure is similar to the one described by Goodnick and Lugli. ${ }^{10}$ The simulations assume initial quasithermal equilibrium followed by a perturbing pulse which removes less than $10 \%$ of the carriers. Under those conditions, we assume that the deviation from the static limit due to dynamical screening is not important.

The general formalism we present is more elaborate than most of the numerous publications in the field. Before it is described and solved, we review briefly the relevant literature. Early numerical results for c-c in a highly excited semiconductor are found in the work of Binder et al. ${ }^{11}$ This cal-

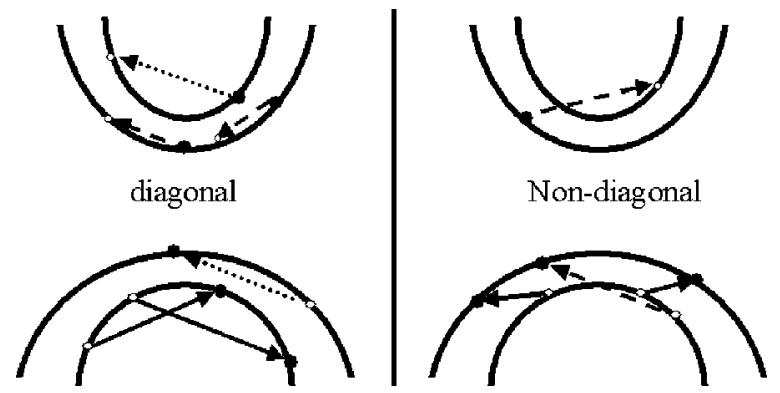

FIG. 1. Schematic diagrams of direct scattering in a multisubband system. Left: three examples of diagonal scattering where carriers are scattered within subbands. Right: two examples of nondiagonal scattering where carriers change subband. 
culation starts from perturbed electron and hole plasma in a bulk semiconductor optical amplifier and yields the evolution of both plasma with the screening treated in the dynamical RPA model. The polarization determines the initial perturbation but is not otherwise included in the equations. In Ref. 11 the authors discuss the relaxation time approximation (RTA), which makes the calculations far simpler and yet quite accurate for the case of small deviations from equilibrium.

Derivation of the semiconductor Bloch equations (SBE's) which are the basis for all relevant solutions, is detailed in Ref. 5 for bulk structures using the density matrix formalism, and an analogous derivation with the nonequilibrium Green's functions is given in Ref. 3. The SBE of a multidimensional nanostructure is described in Ref. 8, and simulations of the electron hole generation rate for bulk semiconductors under the influence of c-c and carrier-phonon scattering within the static screening limit are given in Ref. 7. The effects of Coulomb scattering matrix elements in quantum wells are described in Ref. 12, and an analog situation in quantum wires is presented in Ref. 13. The microscopic theory of VCSEL's was studied in Ref. 14 using Green function formalism with only the first subband at each band being considered. A full density matrix formalism for a dynamically screened potential in a three-dimensional electron gas is presented by Wyld and Fried ${ }^{6}$ while the general single-particle effective Hamiltonian formalism is found in Ref. 15. A study of c-c scattering within the static screening limit along with the polarization at highly excited bulk semiconductors, where the optical pulse evolution is derived dynamically, is presented in Ref. 16. The non-Markovian limit which includes energy-time uncertainty and memory effects is described by Ref. 13 in a quantum wire model. A different point of view on c-c scattering in cases of optical excitation of moderate carrier densities is given in Ref. 17 and in recent publications, ${ }^{18-20}$ where the screening is built self-consistently throughout the propagation of a pulse by applying the Green formalism for the screened Coulomb potential.

This paper is organized as follows. In the next section we outline the general formalism approach. In Sec. III we present the theoretical approach based on the density matrix theory, along with mathematical aspects of the SBE. In Sec. IV we present simulation results of the SBE's, and the conclusions are given in Sec. V. Appendix A contains a derivation of the Coulomb matrix elements in $\mathrm{SCH}$ based on the $\mathbf{k} \cdot \mathbf{p}$ model; also presented are results on the bare and of the static screened Coulomb potential. Appendix B elaborates on the details of the SBE derivation in the density matrix formalism. Appendix $\mathrm{C}$ handles the scattering rate equations and elaborates on mathematical-related issues concerning diagonal and nondiagonal scattering as well as direct versus exchange scattering.

\section{GENERAL FORMALISM}

In order to formulate a model which is applicable to semiconductor quantum wells, we use a general notation where $\mathbf{r}_{\|}$ and $\mathbf{k}$ represent the free-dimension space vector and the single-particle wave vector, respectively. The confined states are localized and are denoted by subband index $\lambda_{i}$ where $\lambda$ $=e, h$, with $z$ taken to be the growth (confined) direction. Before the optical pulse perturbs the system, we assume the system to be in quasithermal equilibrium where the populations of electrons and holes are the Fermi-Dirac distributions. The subsequent dynamics are given by rate equations for the carrier number expectation values, $n_{e_{i}, \mathbf{k}}=\left\langle a_{e_{i}, \mathbf{k}}^{\dagger} a_{e_{i}, \mathbf{k}}\right\rangle$ and $n_{h_{i}, \mathbf{k}}=\left\langle b_{h_{i},-\mathbf{k}}^{\dagger} b_{h_{i},-\mathbf{k}}\right\rangle$ and for the dipole expectation value $p_{e_{i}, h_{i}, \mathbf{k}}=\left\langle b_{h_{i},-\mathbf{k}} a_{e_{i}, \mathbf{k}}\right\rangle$. Here $a_{e_{i}, \mathbf{k}}$ and $a_{e_{i}, \mathbf{k}}^{\dagger}$ are the annihilation and creation operators of a conduction electron in a state given by $\left(e_{i}, \mathbf{k}\right)$, and $b_{h_{i}, \mathbf{k}}$ and $b_{h_{i}, \mathbf{k}}^{\dagger}$ are similarly the annihilation and creation operators of a valence hole in a state $\left(h_{i}, \mathbf{k}\right)$. In the following we shall simply refer to $n_{e_{i}, \mathbf{k}}$ and $n_{h_{i}, \mathbf{k}}$ as subband distributions and to $p_{e_{i}, h_{i}, \mathbf{k}}$ as an intersubband polarization.

The intersubband polarizations of the kind $n_{e_{i}, e_{j}, \mathbf{k}}$ $=\left\langle a_{e_{i}, \mathbf{k}}^{\dagger} a_{e_{j}, \mathbf{k}}\right\rangle$ are assumed to vanish if there is no driving force term in the system Hamiltonian. We also have $n_{e_{i}, \mathbf{k}, \mathbf{p}}$ $=\left\langle a_{e_{i}, \mathbf{k}}^{\dagger} a_{e_{i}, \mathbf{p}}\right\rangle=n_{e_{i}, \mathbf{k}} \delta_{\mathbf{k}, \mathbf{p}}$ due to translational symmetry for the free directions.

In the following, we will assume that intersubband polarizations are dominated by the first transition (that is, the optical pulse transition frequency is resonant only for transitions between the first conduction and first valence subbands, which is a very good approximation for quantum wells). The rate equations for the carrier distributions and polarizations are derived from the operator equations of motion in the Heisenberg picture. They comprise two parts: denoted coherent and collisional. The details of the coherent part are dictated by the noninteracting Hamiltonian, the radiation-matter interaction (optical pulse), and by the lowest-order contribution due to the c-c Coulomb interaction which causes bandgap energy and Rabi frequency renormalizations. It also gives rise to excitonic effects but these are neglected in the regime of very high carrier densities. For the radiation-matter interaction we consider only induced transitions so that spontaneous emission is neglected. The collisional part of the equations is governed by higher-order contributions of the $\mathrm{c}-\mathrm{c}$ Coulomb interaction which give rise to relaxation processes that drive the particle population towards thermal equilibrium. Such relaxation processes (c-c scattering) are divided into particle-conserving processes such as intrasubband thermalization and intraband impact ionization and to non-particle-conserving processes caused by Coulomb -nduced transitions such as Auger recombination and interband impact ionization. The nonconserving processes can be neglected due to their small effect in the time scales where the particle conservation processes make considerable changes in the plasma. The most general formalism takes the form

$$
\begin{gathered}
\frac{d}{d t} n_{\alpha, \mathbf{p}}=\frac{d}{d t} n_{\alpha, \mathbf{p}}\left|c o h+\frac{d}{d t} n_{\alpha, \mathbf{p}}\right|_{c o l}, \\
\frac{d}{d t} p_{\mathbf{p}}=\frac{d}{d t} p_{\mathbf{p}}\left|c o h+\frac{d}{d t} p_{\mathbf{p}}\right|_{c o l},
\end{gathered}
$$


and is also suitable when the Green function notation is employed. The explicit form is the SBE set. In the model we present, these equations have a similar form to those given in Ref. 8 except that it includes exchange scattering processes in the collisional part and it neglects all intrasubband polarizations as well as contributions to interband polarization from other than the first transition.

\section{THEORETICAL MODEL}

In the following analysis, we use the density matrix approach along with the second-quantization ${ }^{21}$ (SQ) representation (Fock representation). The system Hamiltonian which includes noninteracting, radiation-matter interaction and
Coulomb interaction terms is calculated in the SQ representation using standard techniques:

$$
\begin{gathered}
H=H_{N I}+H_{\text {rad-mat }}+H_{C}, \\
H_{N I}=\sum_{\mathbf{k}, e_{i}, h_{i}}\left(\varepsilon_{e_{i}, k} a_{e_{i}, \mathbf{k}}^{\dagger} a_{e_{i}, \mathbf{k}}+\varepsilon_{h_{i}, k} b_{h_{i}, \mathbf{k}}^{\dagger} b_{h_{i}, \mathbf{k}}\right),
\end{gathered}
$$

$$
\begin{aligned}
H_{\text {rad-mat }}= & -\sum_{\mathbf{k}}\left[\mathbf{E}(t) \cdot \boldsymbol{\mu}_{\mathbf{k}} a_{e_{1}, \mathbf{k}}^{\dagger} b_{h_{1},-\mathbf{k}}^{\dagger}\right. \\
& \left.+\mathbf{E}^{*}(t) \cdot \boldsymbol{\mu}_{\mathbf{k}}^{*} b_{h_{1},-\mathbf{k}} a_{e_{1}, \mathbf{k}}\right]
\end{aligned}
$$

$$
\begin{aligned}
H_{C}= & \sum_{\mathbf{k}, \mathbf{k}^{\prime}, \mathbf{q}}\left[-\sum_{e_{i}^{\prime}, h_{j}^{\prime}, h_{j}, e_{i}} V_{q}\left(e_{i}^{\prime}, h_{j}^{\prime} ; h_{j}, e_{i}\right) a_{e_{i}^{\prime}, \mathbf{k}+\mathbf{q}}^{\dagger} b_{h_{j}^{\prime}, \mathbf{k}^{\prime}-\mathbf{q}}^{\dagger} b_{h_{j}, \mathbf{k}^{\prime}} a_{e_{i}, \mathbf{k}}\right. \\
& \left.+\frac{1}{2}\left(\sum_{e_{l}^{\prime}, e_{i}^{\prime}, e_{i}, e_{l}} V_{q}\left(e_{l}^{\prime}, e_{i}^{\prime} ; e_{i}, e_{l}\right) a_{e_{l}^{\prime}, \mathbf{k}+\mathbf{q}}^{\dagger} a_{e_{i}^{\prime}, \mathbf{k}^{\prime}-\mathbf{q}}^{\dagger} a_{e_{i}, \mathbf{k}^{\prime}} a_{e_{l}, \mathbf{k}}+\sum_{h_{l}^{\prime}, h_{j}^{\prime}, h_{j}, h_{l}} V_{q}\left(h_{l}^{\prime}, h_{j}^{\prime} ; h_{j}, h_{l}\right) b_{h_{l}^{\prime}, \mathbf{k}+\mathbf{q}}^{\dagger} b_{h_{j}^{\prime}, \mathbf{k}^{\prime}-\mathbf{q}}^{\dagger} b_{h_{j}, \mathbf{k}^{\prime}} b_{h_{l}, \mathbf{k}}\right)\right],
\end{aligned}
$$

where $\varepsilon_{\lambda_{i}, k}$ denotes the single-particle energy in the state $\left(\lambda_{i}, \mathbf{k}\right), \mathbf{E}(t)$ is the electric field of the optical pulse, and $\mu_{\mathbf{k}}$ is the dipole matrix element. $V_{q}\left(\lambda_{i}, \lambda_{j} ; \lambda_{l}, \lambda_{m}\right)$ denotes the bare Coulomb matrix element, describing the c-c scattering process of two particles from subbands $\lambda_{l}, \lambda_{m}$ to subband $\lambda_{i}, \lambda_{j}$, with $\mathbf{q}$ transferred momentum. Functional derivation and discussion of the bare and static screened Coulomb matrix elements in heterostructures is given in Appendix A.

\section{A. Semiconductor Bloch equations}

A detailed derivation of the SBE's in nanostructures under our approximations is given in Appendix B. A compact formulation of the results comprises three rate equations: two for the electron and hole distributions in a specific subband and one for the interband polarizations induced by the optical transitions, $p_{\mathbf{k}} \equiv p_{e_{1}, h_{1}, \mathbf{k}}$

$$
\begin{gathered}
\frac{d}{d t} n_{e_{i}, \mathbf{p}}=\left.\frac{d}{d t} n_{e_{i}, \mathbf{p}}\right|_{c o l}+i\left[\Omega_{\mathbf{p}} p_{\mathbf{p}}^{*}-\Omega_{\mathbf{p}}^{*} p_{\mathbf{p}}\right] \delta_{e_{i}, e_{1}}, \\
\frac{d}{d t} n_{h_{i}, \mathbf{p}}=\left.\frac{d}{d t} n_{h_{i}, \mathbf{p}}\right|_{c o l}+i\left[\Omega_{\mathbf{p}} p_{\mathbf{p}}^{*}-\Omega_{\mathbf{p}}^{*} p_{\mathbf{p}}\right] \delta_{h_{i}, h_{1}}, \\
\frac{d}{d t} p_{\mathbf{p}}=\left.\frac{d}{d t} p_{\mathbf{p}}\right|_{c o l}-i \omega_{\mathbf{p}} p_{\mathbf{p}}+i \Omega_{\mathbf{p}}\left[1-n_{h_{1}, \mathbf{p}}-n_{e_{1}, \mathbf{p}}\right], \\
\omega_{\mathbf{p}}=\frac{1}{\hbar}\left(\varepsilon_{e_{1}, \mathbf{p}}+\varepsilon_{h_{1}, \mathbf{p}}\right) \\
-\sum_{\mathbf{q}} V_{q}\left(e_{1}, h_{1} ; h_{1}, e_{1}\right)\left(n_{e_{1}, \mathbf{p}+\mathbf{q}}+n_{h_{1}, \mathbf{p}+\mathbf{q}}\right),
\end{gathered}
$$

$$
\Omega_{\mathbf{p}}=\frac{\mathbf{E}(t) \cdot \mu_{\mathbf{p}}}{\hbar}+\frac{1}{\hbar} \sum_{\mathbf{q}} V_{q}\left(e_{1}, h_{1} ; h_{1}, e_{1}\right) p_{\mathbf{p}+\mathbf{q}},
$$

where $\omega_{\mathbf{p}}$, and $\Omega_{\mathbf{p}}$ denote, respectively, the first-order renormalized transition and Rabi frequencies where nondiagonal Coulomb contributions to the renormalized frequencies were neglected due to their relative small effect compared with the diagonal contributions. Equations (6)-(10) are the SBE's for the interacting particles model. Taking the Coulomb terms $V_{q}(\cdots)$ to be zero gives the SBE's in the free-carrier model.

The collisional contribution is

$$
\begin{gathered}
\left.\frac{d}{d t} n_{\alpha_{i}, \mathbf{p}}\right|_{c o l}=\left(\Gamma_{\alpha_{i}, \mathbf{p}}^{\text {in }}+\Delta_{\alpha_{i}, \mathbf{p}}^{\text {in, (2) }}\right)\left(1-n_{\alpha_{i}, \mathbf{p}}\right) \\
-\left(\Gamma_{\alpha_{i}, \mathbf{p}}^{\text {out }}+\Delta_{\alpha_{i}, \mathbf{p}}^{\text {out },(2)}\right) n_{\alpha_{i}, \mathbf{p}} \\
+\left(\Delta_{\alpha, \mathbf{p}}^{(1)} p_{\mathbf{p}}+c . c\right) \delta_{\alpha_{1}, \alpha_{i}} \\
\left.\frac{d}{d t} p_{\mathbf{p}}\right|_{c o l}=\sum_{\alpha_{1}}\left[-\left(\Gamma_{\alpha_{1}, \mathbf{p}}^{\text {in }}+\Gamma_{\alpha_{1}, \mathbf{p}}^{\text {out }}\right) p_{\mathbf{p}}+\Lambda_{\alpha_{1}, \mathbf{p}}^{\text {in, (1) }}\left(1-n_{\alpha_{1}, \mathbf{p}}\right)\right. \\
\left.+\Lambda_{\alpha_{1}, \mathbf{p}}^{\text {out },(1)} n_{\alpha_{1}, \mathbf{p}}+\Lambda_{\alpha_{1}, \mathbf{p}}^{(2)} p_{\mathbf{p}}+\Lambda_{\alpha_{1}, \mathbf{p}}^{(3)}\right]
\end{gathered}
$$

where the superscripts in the brackets of the rates $\Delta$ and $\Lambda$ refer to the polarization power. For relatively weak optical pulses the terms involving $\Delta^{(1)}, \Delta^{(2)}, \Lambda^{(2)}$, and $\Lambda^{(3)}$ are negligible (these involve the square- and third-power polarization), and the equations become the well-known Boltzmann equations for c-c scattering. In all rate terms, exchange contributions are considered. The sum refers to summation 
over first conduction and first valence subbands $\left(\alpha_{1}\right.$ $\left.=e_{1}, h_{1}\right)$ (dominant transition). The explicit form of these rates is given in Appendix C. Figure 1 displays the direct scattering terms of the $\Gamma$ rates.

\section{B. Numerical aspects}

In addition to a detailed physical formalism, the results we present below involve considerable computational complexities. All computer programs used to solve the SBE's were written in $\mathrm{C}++$ and can be described as CPU bounded. A memory-bound program would have consumed memory over and above the RAM limit, which would have caused a bottleneck due to frequent disk access. The calculations were performed using the Origin 2001 at the HPCU center in Tel-Aviv. The simulations used 32 dedicated parallel SGI CPU's, each operating at $500 \mathrm{MHz}$ and having a memory size beyond the program needs. A typical simulation ran for 12 days. For illustration, these simulations would run on a single modern PC for approximately 1 year.

The SBE's given by Eqs. (6)-(12) were solved for a typical SCH QW laser amplifier with the following parameters. The total width of the heterostructure was $985 \AA$, comprising a single $\operatorname{In}_{0.25} \mathrm{Ga}_{0.75}$ As 85 - $\AA$-wide well with $100-\AA \mathrm{GaAs}$ barriers on each side. The rest of the structure comprises $\mathrm{Al}_{0.18} \mathrm{Ga}_{0.82} \mathrm{As}$ on both sides. The temperature was fixed at $300 \mathrm{~K}$ and strain effects were included. The $E-k$ diagrams and the confined wave functions where computed following Ref. 22. The particle masses at the $\Gamma$ point $(\mathbf{k}=0)$ and Coulomb matrix elements were extracted from these data.

$$
\begin{gathered}
m_{e 1}=0.03 m_{0}, \quad m_{h 1}=0.055 m_{0}, \\
m_{e 2}=0.033 m_{0}, \quad m_{h 2}=0.06 m_{0}, \\
E_{e 1}-E_{h 1}=1.2312 \mathrm{eV}, \quad E_{h 2}-E_{h 1}=0.0379 \mathrm{eV}, \\
E_{e 2}-E_{e 1}=0.0709 \mathrm{eV},
\end{gathered}
$$

where all of the energies were computed at the $\Gamma$ point. The medium was excited by a 75-fs Gaussian-shaped optical pulse (the unnormalized Rabi frequency at the peak of the pulse $\mathbf{E}_{0} \cdot \boldsymbol{\mu}_{\mathbf{k}} / \hbar$ was approximately $7.5 \times 10^{12} \mathrm{~Hz}$ ). The frequency of the field was chosen to coincide with the gain region of the first transition $e_{1}-h_{1}$. The amplifier is assumed to be thin; namely, we do not consider pulse propagation effects or any distributed nonlinearities. All simulations consider a parabolic band structure.

Any nonparabolic character of the valence subbands introduces some changes to the relaxation rates, due to differences in the density of states. These effects are considered in a separate paper. ${ }^{23}$ The qualitative impact of c-c scattering in multisubband structures as treated in this paper remains, however, unchanged even in the cases where the density of states is modified.

The calculations we present disregarded interface phonons ${ }^{24}$ whose scattering rates may, in principle, be com-

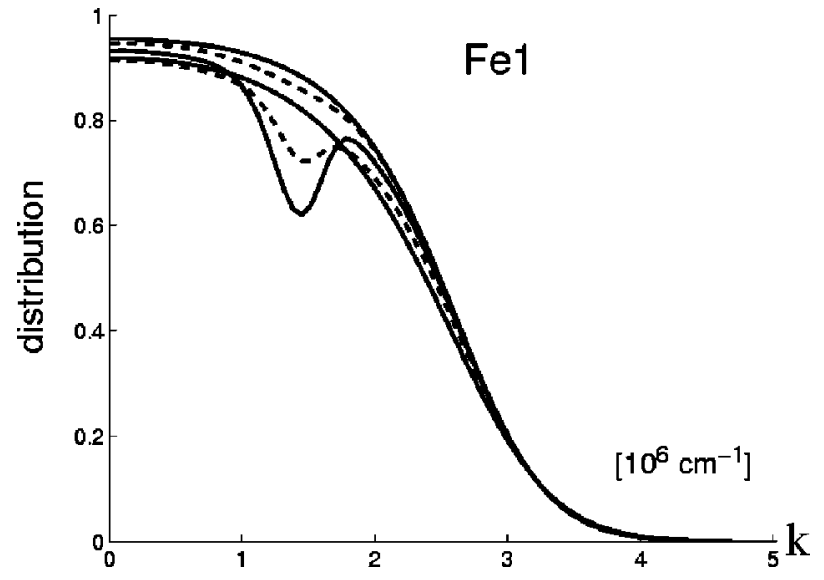

FIG. 2. Evolution of the first conduction-confined subband distribution. Upper solid line denotes the initial distribution $-\infty$ fs before the arrival of the pulse peak. Upper dashed line denotes the distribution at $-40 \mathrm{fs}$, lower solid line $+80 \mathrm{fs}$ at which the plasma is the most perturbed, lower dashed line $+250 \mathrm{fs}$, and middle solid line $+1.2 \mathrm{ps}$.

parable with those originating from $\mathrm{c}-\mathrm{c}$ scattering in multiple-quantum-well (MQW) structures with relatively narrow wells $(\sim 50 \AA) .{ }^{25-27}$ These calculations do not consider screening, however, which tends to decrease the scattering rates.

The heterostructure we consider comprises a single $85-\AA$ quantum well whose hole wave functions (first and second subband) and electron wave function (first subband) are mainly localized at the well center. This results in a poor overlap of carrier and IP envelope functions, which in turn reduces the rate. Intrasubband scattering rates due to IP's at the second conduction subband may be comparable with the c-c time constants under consideration. However, the finite confinement of electrons in the well, which in the present case is of the order of $100 \mathrm{meV}$, reduces the rate of the latter.

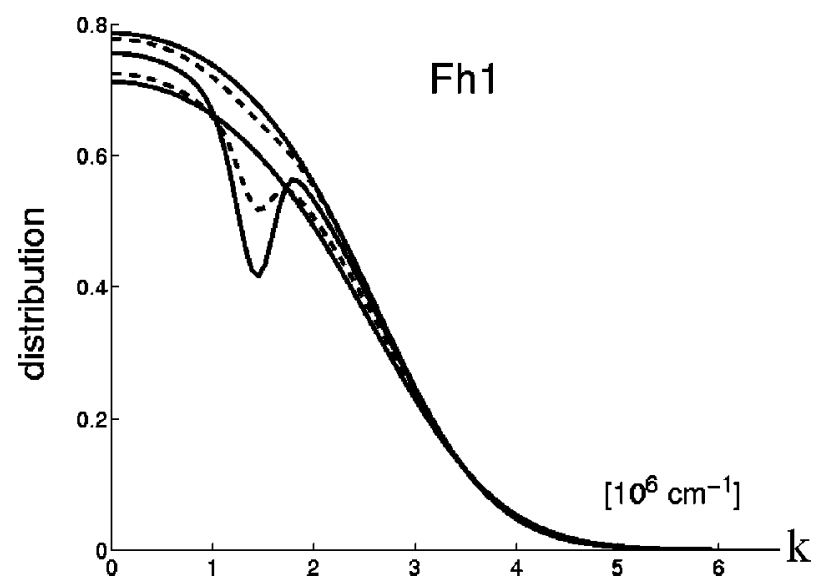

FIG. 3. Evolution of the first valence-confined subband distribution. Upper solid line denotes the initial distribution $-\infty$ fs before the arrival of the pulse peak. Upper dashed line denotes the distribution at $-40 \mathrm{fs}$, lower solid line $+80 \mathrm{fs}$ at which the plasma is the most perturbed, lower dashed line $+250 \mathrm{fs}$, and middle solid line +1.2 ps. 


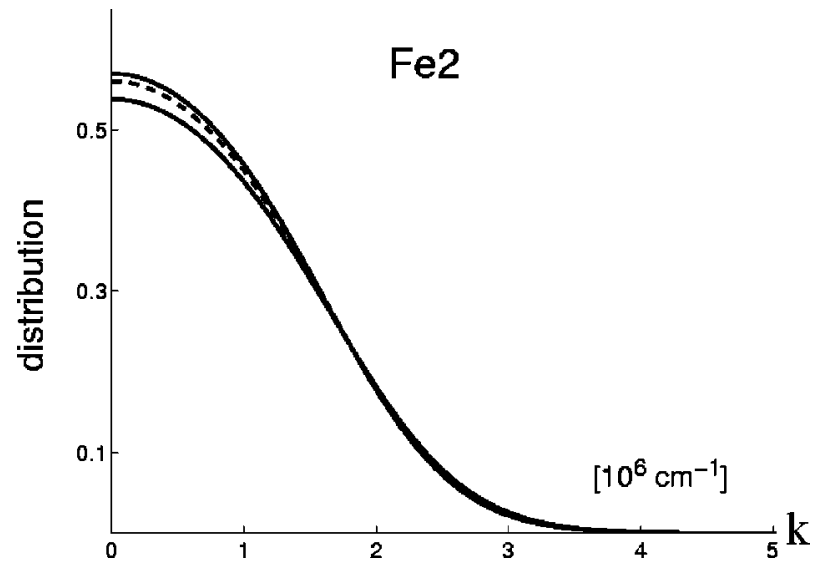

FIG. 4. Evolution of the second conduction-confined subband distribution. Upper solid line denotes the initial distribution $-\infty$ fs before the arrival of the pulse peak. Dashed line denotes the distribution at $+250 \mathrm{fs}$, lower solid line $+1.2 \mathrm{ps}$.

Intersubband transition rates (analog to nondiagonal scattering) caused by IP's are negligible in single-quantum-well structures.

Our first set of simulations considered multisubband parabolic energy-wave-vector dispersion. At "infinite time," before the arrival of the pulse peak, the polarization inside the medium is assumed to be zero throughout and the initial subband distributions are quasithermal Fermi-Dirac distributions within the bands. At $300 \mathrm{~K}$ and for an exemplary total carrier density of $N_{2 d} \sim 1.3 \times 10^{12} \mathrm{~cm}^{-2}$, we extracted the chemical potential at each band and calculated the various densities which are $N_{2 d}^{e_{1}} \approx 0.76 N_{2 d}, \quad N_{2 d}^{h_{1}} \approx 0.7 N_{2 d}, \quad N_{2 d}^{e_{2}}$ $\approx 0.24 N_{2 d}$, and $N_{2 d}^{h_{2}} \approx 0.3 N_{2 d}$. The densities of higher subbands $\left(e_{3}, h_{3}, \ldots\right)$ were found to be negligible.

The second set of simulations considered only one subband within each band and use the same parameters as before only with densities of $N_{2 d}^{e_{1}}=N_{2 d}^{h_{1}}=N_{2 d}=10^{12} \mathrm{~cm}^{-2}$.

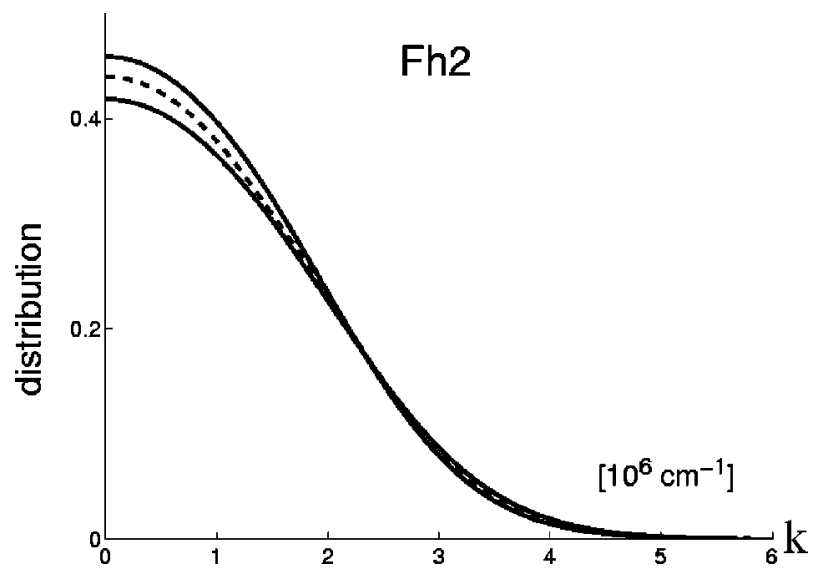

FIG. 5. Evolution of the second valence-confined subband distribution. Upper solid line denotes the initial distribution $-\infty$ fs before the arrival of the pulse peak. Dashed line denotes the distribution at $+250 \mathrm{fs}$, lower solid line $+1.2 \mathrm{ps}$.

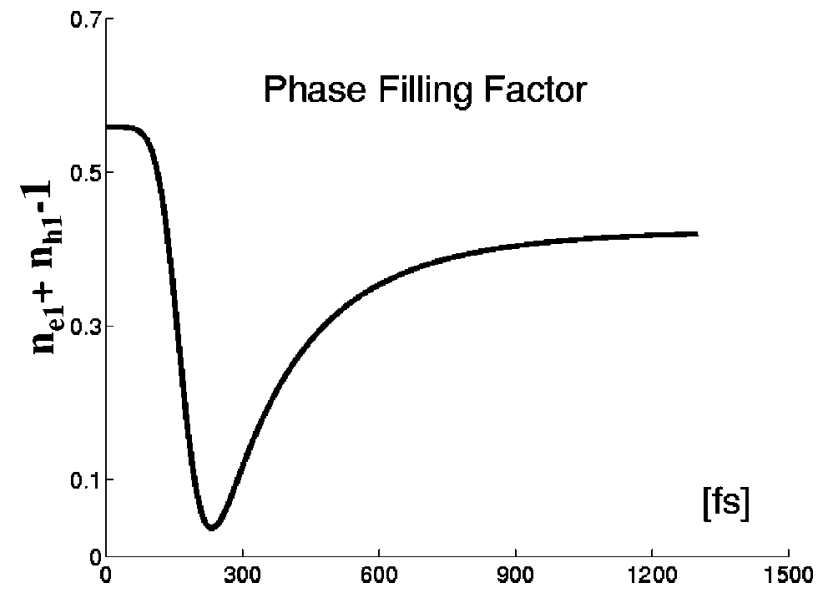

FIG. 6. Phase filling factor at the momentum region where the plasma is most perturbed.

\section{RESULTS AND DISCUSSION}

The following figures show calculated time evolutions of each of the subband distributions, according to Eqs. (6)(12). Figures 2 and 3 describe the behavior of the first subband in the conduction and valence bands, respectively. Each figure comprises five curves describing the distributions at times the following: $-\infty$ fs, upper solid line; -40 fs, upper dashed line; $80 \mathrm{fs}$, lower solid line; $250 \mathrm{fs}$, lower dashed line; and $1.2 \mathrm{ps}$, middle solid line. The reference time $t=0 \mathrm{fs}$ is taken at the peak of the arriving pulse. Figures 4 and 5 are similar but address the evolution of the second subband of each band, which in the present model are assumed not to participate in the optical transition. The distributions of these subbands are denoted by three curves which refer the following: to $-\infty \mathrm{fs}$, upper solid line; $250 \mathrm{fs}$, dashed line; $1.2 \mathrm{ps,}$ lower solid line.

The interaction with the optical pulse removes carriers from the gain region at energies below the average particle energy in the plasma. Hence, the plasma heats and gain phase filling factor $\left(n_{e_{1}, k}+n_{h_{1}, k}-1\right)$ do not relax into their initial condition but rather into a new, lower value which decreases the gain. Figure 6 describes the phase filling factor

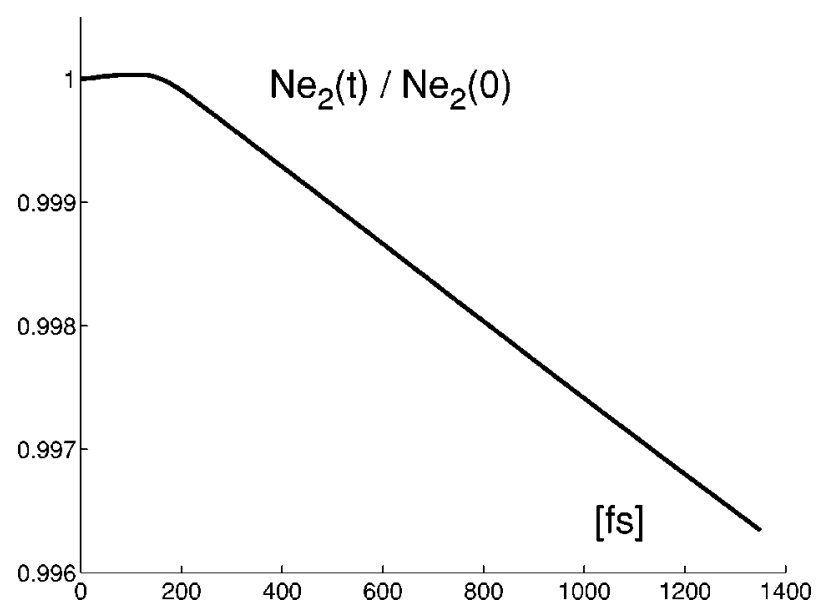

FIG. 7. Normalized total density at the second conductionconfined subband. 


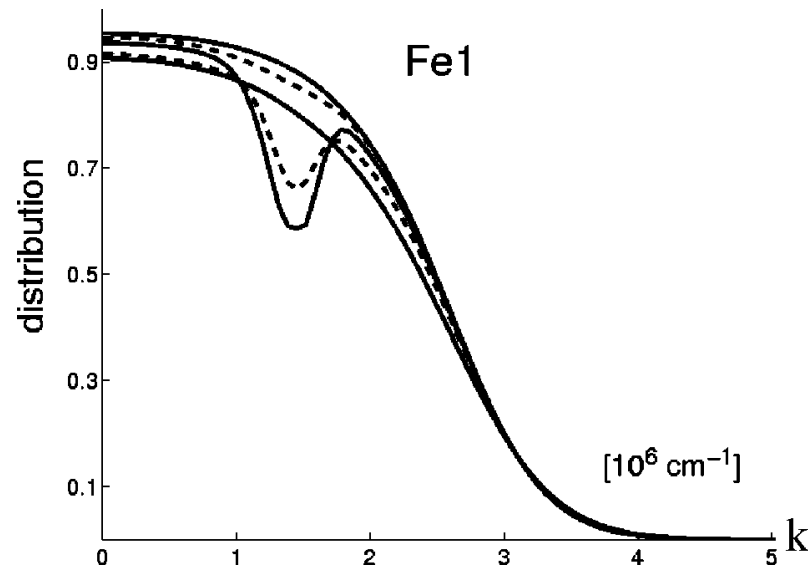

FIG. 8. Evolution of the first conduction-confined subband distribution. Upper solid line denotes the initial distribution $-\infty$ fs before the arrival of the pulse peak. Upper dashed line denotes the distribution at $-40 \mathrm{fs}$, lower solid line $+80 \mathrm{fs}$ at which the plasma is the most perturbed, lower dashed line $+250 \mathrm{fs}$, and middle solid line $+1.2 \mathrm{ps}$.

at the momentum region where the plasma is most perturbed (at this figure the $t=0$ fs refers to -150 fs before the peak arrival). The increase shown in the figure means that $80 \mathrm{fs}$ after the peak of the pulse, the effect of the c-c scattering mechanism already dominates over the effect of the tail of the optical pulse and causes the phase filling factor to increase. Approximately $500 \mathrm{fs}$ after the pulse peak, the phase filling factor stabilizes except for a continuous mild increase which continues due to thermalization processes as well as a transfer of particles from the second subbands at each band to the first. After a significantly longer time, on the order of a few ps, all of the subbands reach new quasi-Fermi-Dirac distributions at the same temperature. Figure 7 describes the time dependence of the distribution in the second conduction subband normalized to its initial value. As long as the per-

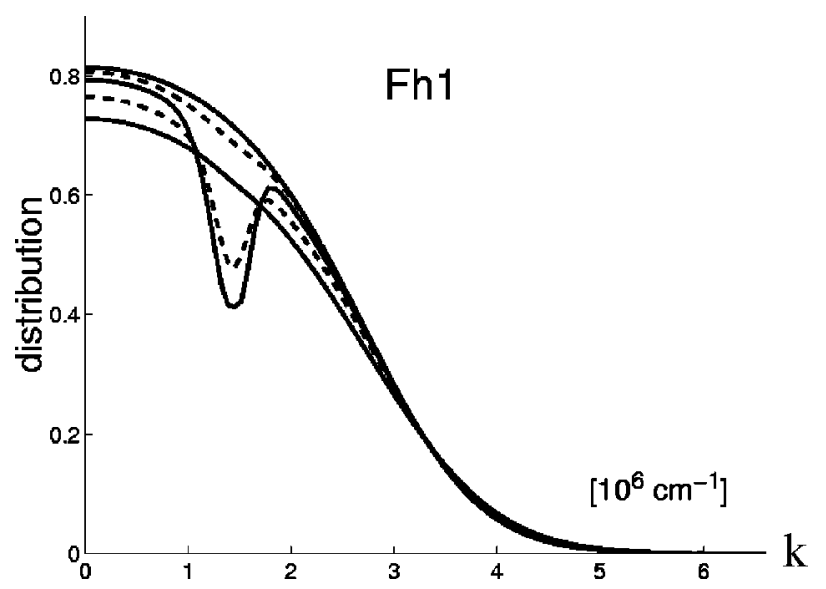

FIG. 9. Evolution of the first valence-confined subband distribution. Upper solid line denotes the initial distribution $-\infty$ fs before the arrival of the pulse peak. Upper dashed line denotes the distribution at $-40 \mathrm{fs}$, lower solid line $+80 \mathrm{fs}$ at which the plasma is the most perturbed, lower dashed line $+250 \mathrm{fs}$, and middle solid line +1.2 ps.

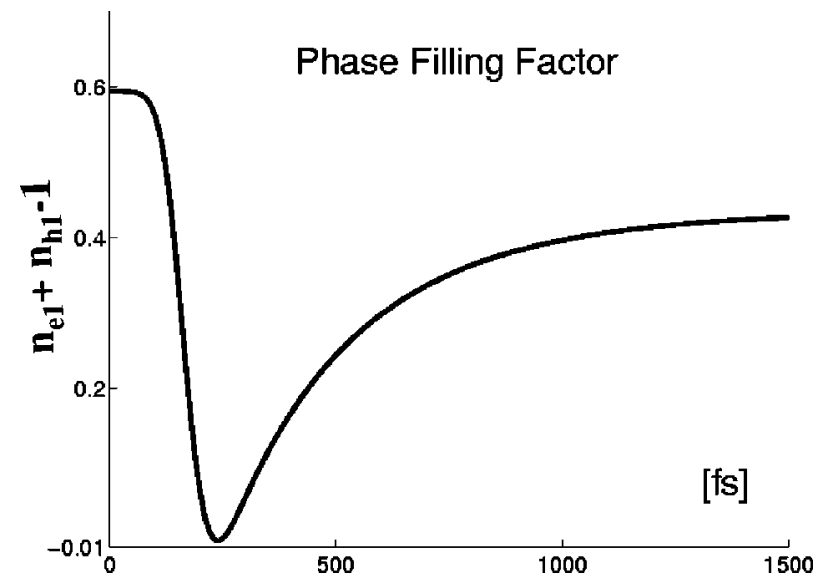

FIG. 10. Phase filling factor at the momentum region where the plasma is most perturbed in the simulation without the second subband.

turbation due to the optical pulse has a stronger effect than the c-c scattering process, the distribution remains approximately constant. At later times, when the c-c scattering process mediates the transfer of particles to the first subband, the normalized distribution naturally decreases monotonically. This decrease can be approximated in the RTA as $N_{e_{2}}(t)$ $\simeq N_{e_{2}}(0) \exp (-t / \tau)$, where $\tau=300$ ps.

The calculation shows that the process transfers $0.4 \%$ of the second subband population into the first subband within 1.2 ps after the pulse peak. For comparison, in the most perturbed case, the optical pulse removes $7.5 \%$ of the first subband population. Using the RTA as before, it would take about 20 ps to replenish the original population of the first subband.

In order to further highlight the significance of including more than one subband in the calculation, we recalculated the response of the same SCH QW medium with identical physical parameters $\left(T=300 \mathrm{~K}, N_{2 d}^{e_{1}}=N_{2 d}^{h_{1}}=10^{12} \mathrm{~cm}^{-2}\right.$, $\left.m_{e 1}=0.03 m_{0}, \quad m_{h 1}=0.055 m_{0}, \quad E_{e 1}-E_{h 1}=1.2312 \mathrm{eV}\right)$, except that only a single subband was included in each band.

TABLE I. Final distributions (at 1.2 ps) of Figs. 2-5, 8, and 9 approximated as Fermi-Dirac distributions. The chemical potential and temperatures differences between these distributions and of their respective initial distributions are given.

\begin{tabular}{lcc}
\hline \hline Subband & $\Delta u(\mathrm{meV})$ & $\Delta T(\mathrm{~K})$ \\
\hline$e_{1}{ }^{\mathrm{a}}$ & -7.1 & 40 \\
$h_{1}{ }^{\mathrm{a}}$ & -6.8 & 40 \\
$e_{2}{ }^{\mathrm{a}}$ & -2.3 & 20 \\
$h_{2}{ }^{\mathrm{a}}$ & -5.1 & 37 \\
$e_{1}{ }^{\mathrm{b}}$ & -8 & 50 \\
$h_{1}{ }^{\mathrm{b}}$ & -7.4 & 53 \\
\hline \hline
\end{tabular}

${ }^{a}$ Multisubband simulation.

${ }^{\mathrm{b}}$ Single subband was included in each band. 
Figures 8 and 9 exhibit similar curves as in Figs. 2 and 3. Comparing the the lower dashed lines which denote the subband distribution $250 \mathrm{fs}$ after the arrival of the pulse peak, it is obvious that including the second subband in the calculations yields a significantly faster recovery of the kinetic hole.

Comparing the phase filling factors, Figs. 6 and 10 show two clear differences. First, the minimum value reached due to saturation in the multisubband model is higher than the transparency point (where the induced emission and absorption are equal), while in the single-subband model, the saturation is sufficiently large to reach the transparency point. Second, the recovery of the phase filling factor is faster in the multisubband model as can be deduced by observation of the shape of the respective curves.

By assuming that the final distributions (at $1.2 \mathrm{ps)} \mathrm{of} \mathrm{Figs.}$ $2-5,8$, and 9 are given by Fermi-Dirac distributions we have extracted the chemical potential and temperature of each subband out of the first and third wave-vector moments (correspond for the total density and energy of each subband). The fitting between the approximated Fermi-Dirac distributions and the final distributions was excellent. The chemical potential and temperature differences between the initial distributions (at $t=-\infty \mathrm{ps)}$ and the extrapolated final distributions are given in Table I. As can be seen from the table, the perturbed subbands $\left(e_{1}\right.$ and $\left.h_{1}\right)$ of the multisubband simulation reach a lower temperature than in the second simulation where only one subband at each band was considered $(340 \mathrm{~K}$ versus $350 \mathrm{~K}$ ), the respective chemical potentials are also less perturbed. An additional interesting feature is that due to the fact that in this specific considered example the diagonal Coulomb matrix element of the second conduction subband is relatively weaker than the other subband diagonal elements (see Appendix A), the $e_{2}$ subband was less perturbed than the other subbands, which can be seen from the smaller deviation of the chemical potential and temperature. We have not taken carrier-phonon interactions into account, which would have relaxed the carrier temperature to the lattice temperature within a relaxation time of the order $0.6 \mathrm{ps}$.

One more aspect of the improvement gained in calculating the recovery of a spectral hole with the multisubband formalism is shown in Fig. 11. The figure compares the time evolution of the absolute value of the polarization at the momentum region where the plasma is most perturbed calculated with one and with two subbands. The dashed line, which denotes the single-subband case, reaches higher values than the corresponding multisubband case. Coulomb scattering which involves higher subbands adds a contribution to the thermalization of the first subbands moderating the increase in the value of the polarization. Consequently, the dephasing is stronger.

Considering a simple phenomenological model without high-order polarization terms in the collisional part and polarization transfer of linear terms, the polarization dephasing is given by ${ }^{15}$

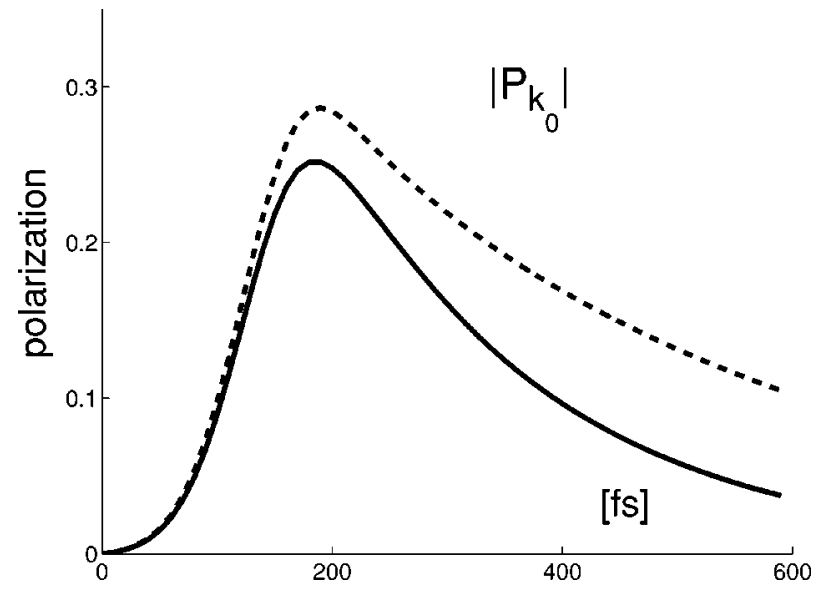

FIG. 11. The absolute value of the polarization vs time. The polarization considered here is extracted from the momentum region where the plasma is most perturbed. The lower solid line denotes the multisubband case whereas the upper dashed line denotes the nonmultisubband case.

$$
\begin{gathered}
\left.\frac{d n_{\alpha_{1}, \mathbf{k}}}{d t}\right|_{c o l}=\Gamma_{\alpha_{1}, \mathbf{k}}^{i n}\left(1-n_{\alpha_{1}, \mathbf{k}}\right)-\Gamma_{\alpha_{1}, \mathbf{k}}^{o u t} n_{\alpha_{1}, \mathbf{k}}, \\
\left.\frac{d p_{\mathbf{k}}}{d t}\right|_{c o l}=-\gamma_{\mathbf{k}} p_{\mathbf{k}}, \\
\gamma_{\mathbf{k}}=\Gamma_{e_{1}, \mathbf{k}}^{i n}+\Gamma_{e_{1}, \mathbf{k}}^{o u t}+\Gamma_{h_{1}, \mathbf{k}}^{\text {in }}+\Gamma_{h_{1}, \mathbf{k}}^{\text {out }},
\end{gathered}
$$

where the $\Gamma$ are the same as in Appendix C. The dephasing, which is responsible for the decay of the polarization as well as for its suppression during the polarization buildup, is clearly stronger in the multisubband case which implies that its $\Gamma$ rates are stronger, where these terms are directly caused by the c-c Coulomb scattering.

\section{CONCLUSIONS}

This paper described a detailed calculation of the c-c scattering contribution to a spectral hole recovery in a roomtemperature inverted QW gain medium under the parabolic band assumption and using the density matrix formalism. The calculations were performed for the high-injection regime typical of semiconductor optical amplifiers. The main contribution of this paper is the inclusion of two subbands in each band while maintaining all exchange terms. A general formalism and the results of elaborate numerical calculations have been presented. The addition of a second subband in each band has a significant influence on several aspects. First, the recovery of the kinetic hole is measurably faster in the multisubband case when compared to the conventional single-subband formalism. Moreover, following a perturbing optical pulse in the multisubband case, the degree of saturation of the phase filling factor (which is directly related to the gain) is reduced as is the maximum absolute value of the polarization, both due to Coulomb c-c scattering events involving the second subband. The calculation also yields the time evolution of the second subband distribution which clearly shows its contribution to the overall recovery. The 
results obtained from the calculations serve two purposes. Including the second subband clearly leads to a more accurate description of this widely researched problem and, at the same time, the moderation of the role played by spectral hole burning has an important practical impact on the gain nonlinearity of QW lasers and amplifiers and hence on their modulation and switching capabilities.

\section{ACKNOWLEDGMENTS}

H.D. acknowledges financial support of Levi Eshkol and Vatat graduate student grants. The authors thank Professor Amiram Ron for many useful discussions.

\section{APPENDIX A: COULOMB SCATTERING}

In the SQ representation, the wave functions are replaced by field creation and annihilation operators $\Psi^{\dagger}(\mathbf{r})$ and $\Psi(\mathbf{r})$. Using the mode representation in the electron-hole picture, the annihilation operator can be written as

$$
\Psi(\mathbf{r}, t)=\sum_{e_{i}, h_{j}, \mathbf{k}} \Phi_{e_{i}, \mathbf{k}}\left(\mathbf{r}_{\|}, z\right) a_{e_{i}, \mathbf{k}}(t)+\Phi_{h_{j}, \mathbf{k}}\left(\mathbf{r}_{\|}, z\right) b_{h_{j}, \mathbf{k}}^{\dagger}(t)
$$

where the general form of the wave function is given by

$$
\Phi_{\lambda_{l}, \mathbf{k}}\left(\mathbf{r}_{\|}, z\right)=\frac{1}{\sqrt{A}} \sum_{n} e^{i \mathbf{k} \cdot \mathbf{r}_{\|}} \phi_{\mathbf{k}, n}^{\lambda_{l}}(z) u_{\mathbf{k}, n}(\mathbf{r}) .
$$

Here $n$ denotes the band index, $u_{\mathbf{k}, n}(\mathbf{r})$ is the lattice periodic Bloch function of band $n, \phi_{\mathbf{k}, n}^{\lambda_{l}}(z)$ denotes the confined part of the envelope function, and $A$ is the area of the quantum well. Calculations of the single-particle noninteracting $E-k$ dispersion curve, as well as the confined part of the envelope function, were made by following the $\mathbf{k} \cdot \mathbf{p}$ method of Gershoni et $a .^{22}$ They used eight zone center Bloch waves $(|S \downarrow\rangle,|X \downarrow\rangle,|Y \downarrow\rangle,|Z \downarrow\rangle,|S \uparrow\rangle,|X \uparrow\rangle,|Y \uparrow\rangle,|Z \uparrow\rangle)$ with the confined part of the envelope given as

$$
\phi_{\mathbf{k}, n}^{\lambda_{m}}(z)=\frac{1}{\sqrt{(} L_{z}} \sum_{l=-J}^{l=+J} F_{\mathbf{k}, n}^{\lambda_{m}}(l) e^{j\left(2 \pi l / L_{z}\right) z}
$$

Here $L_{z}$ denotes the width of the separate confinement heterostructure, taken to be sufficiently wide to include the tails of bound wave functions. Despite the fact that each wave function is governed by projections of all eight Bloch wave functions (valence and conduction) we label the $e_{i}\left(h_{j}\right)$ if the energy state is above (below) the conduction (valence) edge.

In order to calculate the Coulomb matrix elements we have used the following approximations. First, the Coulomb potential will have the same functional form throughout the heterostructure, even thought the dielectric constant varies from one region of the structure to another. This assumption is based on the fact that the variations of the dielectric constant are quite small (i.e., $\left|\epsilon_{b 1}-\epsilon_{b 2}\right| \ll \epsilon_{b 1}, \epsilon_{b 2}$ ). Second, the $k$ dependence of the confined part of the envelope $\phi_{\mathbf{k}, n}^{\lambda_{l}}(z)$ in the wave function is considered to be weak and can therefore be neglected. This assumption simplifies the algebra a great deal; we shall use the simpler notation $\phi_{n, \lambda_{l}}(z) \equiv \phi_{\mathbf{k}, n}^{\lambda_{l}}(z)$. Third, the $\mathbf{k}$ dependence of the Bloch part $u_{\mathbf{k}, n}\left(\mathbf{r}_{\|}, z\right)$ in the wave function is considered to be weak in the zone center region; therefore, the Bloch functions are calculated at the $\boldsymbol{\Gamma}$ point $(\mathbf{k}=0)$. Fourth, except for the Bloch part in the wave function, the other parts are considered to be constant within the regions of a unit cell, and therefore the integration will be made in two steps: first integration over the volume of a unit cell which involves only the Bloch functions and then throughout the entire volume with the other parts of the wave functions. Applying the above approximations we can calculate Coulomb matrix elements by solving the integral in Eq. (A4). Going to the right-hand side (RHS) of Eq. (A4) requires the substitution of variables and Bessel function identities: $q=\left|\vec{k}_{2}-\vec{k}_{2}^{\prime}\right|=\left|\vec{k}_{1}-\vec{k}_{1}^{\prime}\right|$, and $\delta$ is the Kronecker delta function:

$$
\begin{aligned}
V_{q}\left(\lambda_{l}^{\prime}, \lambda_{m}^{\prime} ; \lambda_{m}, \lambda_{l}\right)= & \sum_{n_{1}, n_{2}} \frac{e^{2}}{A^{2} \epsilon_{b}} \int_{0}^{L_{z}} \int_{0}^{L_{z}} d z_{2} d z_{1} \iint d^{2} \rho_{2} d^{2} \rho_{1} \frac{1}{\sqrt{\left(\vec{\rho}_{2}-\vec{\rho}_{1}\right)^{2}+\left(z_{2}-z_{1}\right)^{2}}} \\
& \times e^{j\left[\left(\mathbf{k}_{1} \cdot \boldsymbol{\rho}_{1}-\mathbf{k}_{1}^{\prime} \cdot \boldsymbol{\rho}_{1}\right)+\left(\mathbf{k}_{2} \cdot \boldsymbol{\rho}_{2}-\mathbf{k}_{2}^{\prime} \cdot \boldsymbol{\rho}_{2}\right)\right]} \phi_{n_{1}, \lambda_{m}^{\prime}}^{*}\left(z_{1}\right) \phi_{n_{1}, \lambda_{m}}\left(z_{1}\right) \phi_{n_{2}, \lambda_{l}^{\prime}}^{*}\left(z_{2}\right) \phi_{n_{2}, \lambda_{l}}\left(z_{2}\right) \\
= & \delta_{\mathbf{k}_{1}+\overrightarrow{\mathbf{k}}_{2}, \overrightarrow{\mathbf{k}}_{1}^{\prime}+\overrightarrow{\mathbf{k}}_{2}^{\prime}} \frac{2 \pi e^{2}}{\epsilon_{b} A q} \sum_{n_{1}, n_{2}} \int_{-L_{z}}^{L_{z}} d z e^{-q|z|} \int_{|z| / 2}^{L_{z}-|z| / 2} d z_{+} \phi_{n_{1}, \lambda_{m}^{\prime}}^{*}\left(z+\frac{z}{2}\right) \phi_{n_{1}, \lambda_{m}} \\
& \times\left(z_{+}-\frac{z}{2}\right) \phi_{n_{2}, \lambda_{l}^{\prime}}^{*}\left(z_{+}+\frac{z}{2}\right) \phi_{n_{2}, \lambda_{l}}\left(z_{+}+\frac{z}{2}\right)
\end{aligned}
$$




$$
\begin{aligned}
& V_{q}\left(\lambda_{l}^{\prime}, \lambda_{m}^{\prime} ; \lambda_{m}, \lambda_{l}\right)=\frac{4 \pi e^{2}}{V \epsilon_{b}} \sum_{\substack{j_{1}, j_{1}^{\prime}, j_{2}, j_{2}^{\prime}, n_{1}, n_{2}}} F_{n_{2}, \lambda_{l}^{\prime}}^{*}\left(j_{2}^{\prime}\right) F_{n_{1}, \lambda_{m}^{\prime}}^{*}\left(j_{1}^{\prime}\right) F_{n_{1}, \lambda_{m}}\left(j_{1}\right) F_{n_{2}, \lambda_{l}}\left(j_{2}\right) \\
& \times\left\{\begin{array}{l}
\frac{1}{q^{2}+\left(\frac{\pi \Delta}{L_{z}}\right)^{2}}-\frac{1-e^{-q L_{z}}}{q L_{z}} \frac{\left[q^{2}-\left(\frac{\pi \Delta}{L_{z}}\right)^{2}\right]}{\left[q^{2}+\left(\frac{\pi \Delta}{L_{z}}\right)^{2}\right]^{2}}, \\
-\frac{1-e^{-q L_{z}}}{q L_{z}} \frac{\left[q^{2}+\left(\frac{\pi \Delta_{+}}{L_{z}}\right)^{2}-\left(\frac{\pi \Delta}{L_{z}}\right)^{2}\right]}{\left[q^{2}+\left(\frac{\pi \Delta+}{L_{z}}\right)^{2}-\left(\frac{\pi \Delta}{L_{z}}\right)^{2}\right]^{2}+\left(\frac{2 \pi \Delta q}{L_{z}}\right)^{2}},
\end{array}\right.
\end{aligned}
$$

where $\Delta=j_{2}+j_{1}^{\prime}-j_{1}-j_{2}^{\prime}$ and $\Delta_{+}=j_{2}-j_{1}^{\prime}+j_{1}-j_{2}^{\prime}$. Here $V$ is the volume of integration. It can be seen from the above equation that the following identities are valid:

$$
\begin{aligned}
V_{q}\left(\lambda_{j}^{\prime}, \lambda_{i}^{\prime} ; \lambda_{i}, \lambda_{j}\right) & =V_{q}\left(\lambda_{i}^{\prime}, \lambda_{j}^{\prime} ; \lambda_{j}, \lambda_{i}\right)=V_{q}^{*}\left(\lambda_{i}, \lambda_{j} ; \lambda_{j}^{\prime}, \lambda_{i}^{\prime}\right) \\
& =V_{q}^{*}\left(\lambda_{j}, \lambda_{i} ; \lambda_{i}^{\prime}, \lambda_{j}^{\prime}\right) .
\end{aligned}
$$

Replacing the bare Coulomb potential $\left[v(r)=e^{2} / \epsilon_{b}|\mathbf{r}|\right]$ in Eq. (A4) with the Yukawa potential $\left[v_{s}(r)\right.$ $\left.=\left(e^{2} / \epsilon_{b}|\mathbf{r}|\right) e^{-\kappa|\mathbf{r}|}\right]$ where $\kappa$ denotes the inverse screening length yields nonanalytical results. Further discussion of the derivation of this potential in the Thomas-Fermi screening model or in the static limit of the RPA or PPA (plasmon pole approximation) for cases of $2 \mathrm{D}$ and $3 \mathrm{D}$ is given in Ref. 28. For the example introduced at the beginning of Sec. III B we have calculated the bare Coulomb matrix elements from the analytic expression (A5), and the static screened elements were numerically computed via Eq. (A4) including the screening factor $\exp (-\kappa|\mathbf{r}|)$ in the integrand.

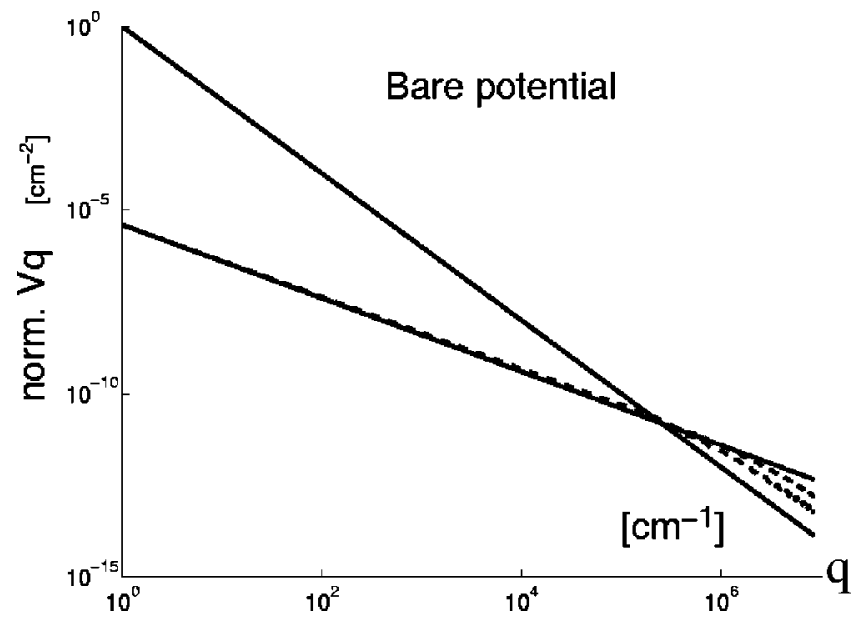

FIG. 12. Diagonal bare Coulomb matrix elements vs momentum transfer $q$. Upper (lower) solid lines denote $V_{q}^{3 d}\left(V_{q}^{2 d}\right)$. Upper dashed curve denotes diagonal matrix element for scattering between particles in the subbands $e_{1}, h_{1}, h_{2}$. Lower dashed curve is for scattering involving the second conduction subband.
Assuming inversion symmetry for our example the only nonvanishing Coulomb terms are diagonal elements of the form $V\left(\alpha_{i}, \alpha_{j} ; \alpha_{j}, \alpha_{i}\right)$ or $V\left(\alpha_{i}, \beta_{j} ; \beta_{j}, \alpha_{i}\right)$ where $\{\alpha, \beta\}$ $=\{e, h\}$ or $\{h, e\}$, nondiagonal elements which involve scattering within the same band such as $V\left(\alpha_{i}, \alpha_{i} ; \alpha_{j}, \alpha_{j}\right)$ or $V\left(\alpha_{i}, \alpha_{j} ; \alpha_{i}, \alpha_{j}\right)$, with $\alpha=\{e, h\}$ and $i \neq j$, and also nondiagonal elements which involve scattering of electrons and holes such as $V\left(\alpha_{i}, \beta_{m} ; \beta_{n}, \alpha_{j}\right)$ with $\{\alpha, \beta\}=\{e, h\}$ or $\{h, e\}$ and where both $i \neq j$ and $m \neq n$ are satisfied. Terms of the kind $V\left(\alpha_{i}, \alpha_{i} ; \alpha_{i}, \alpha_{m}\right)$ or $V\left(\alpha_{i}, \beta_{m} ; \beta_{m}, \alpha_{l}\right)$ are negligible. $^{13}$

Due to the geometry of our example and its physical parameters, the confined part of the envelope functions (A3) of the first two valence subbands as well as the first conduction band are strongly confined to the well region. We can therefore expect that Coulomb matrix elements for scattering between particles in these bands will be closer to the case of pure 2D scattering than matrix elements for scattering that involves the second conduction band. This is confirmed by

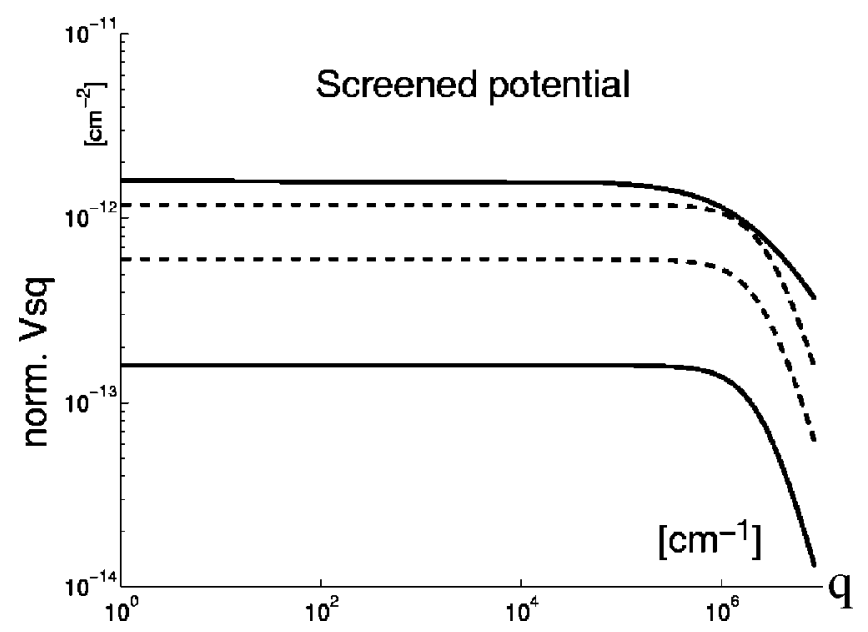

FIG. 13. Diagonal static screened Coulomb matrix elements vs momentum transfer. Upper (lower) solid curve denotes $V_{s, q}^{2 d}\left(V_{s, q}^{3 d}\right)$. Upper dashed curve denotes diagonal matrix element for scattering between particles in the subbands $e_{1}, h_{1}, h_{2}$. Lower dashed curve is for scattering involving the second conduction subband. 


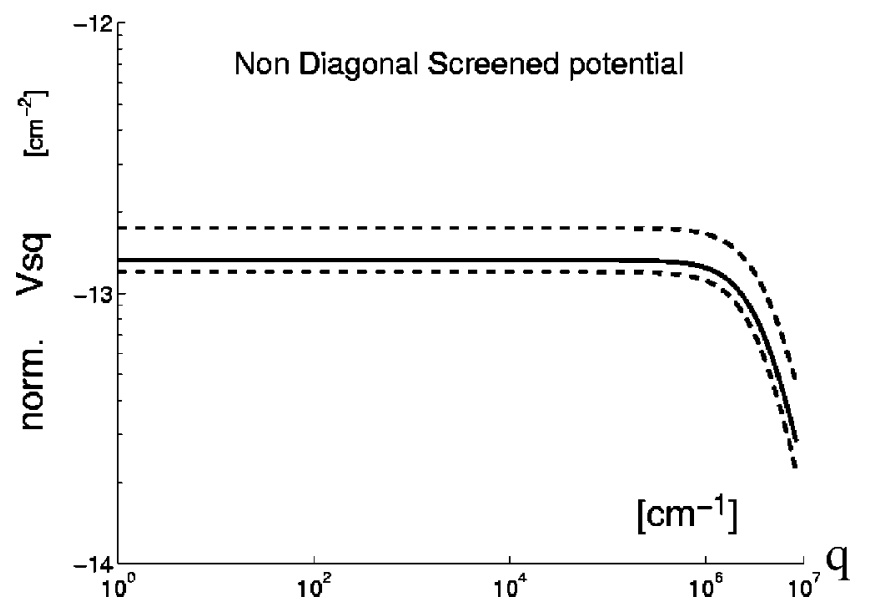

FIG. 14. Nondiagonal static screened Coulomb matrix elements vs momentum transfer $q$. Upper (lower) dashed line shows nondiagonal matrix elements for scattering of valence (conduction) subband carriers. The solid line shows a typical nondiagonal matrix element which comprise a mixture of valence and conduction subband carriers (see text).

Figs. 12 and 13 which display the diagonal bare Coulomb matrix elements $V_{q}$ and diagonal static screened Coulomb matrix elements $V_{s, q}$ as a function of transverse momentum $q$. The matrix elements are divided by $\left(4 \pi^{2} e^{2}\right) /\left(V \epsilon_{b}\right)$; the units of the normalized elements are $\left[\mathrm{cm}^{-2}\right]$. In bulk materials the bare Coulomb matrix element scales as $V_{q}^{3 d} \propto 1 / q^{2}$ while in a pure 2D system it scales as $V_{q}^{2 d} \propto 1 / q$. The curves in Fig. 12 for bulk 3D and pure 2D structures are therefore straight lines (solid) in the double-logarithmic plot. The other curves are diagonal elements where the upper dashed curve shows a typical element for bare Coulomb scattering where none of the particles are from the subband $e_{2}$. The lower dashed curve denotes a typical element where $e_{2}$ is involved. The behavior in the long-wavelength region $q \rightarrow 0$ is close to the purely 2D, while in the $q>10^{6} \mathrm{~cm}^{-1}$ range, it begins to tend toward $3 \mathrm{D}$ behavior.
The screening length used in our calculations was taken to be $\kappa=2.5 \times 10^{6} \mathrm{~cm}^{-1}$, which is consistent with a $2 \mathrm{D}$ carrier density of $10^{12} \mathrm{~cm}^{-2}$. In Fig. 13 the screened Coulomb scattering therefore shows a behavior similar to the bare Coulomb scattering for $q>10^{6} \mathrm{~cm}^{-1}$ but the elements approach constants for $q$ well below $10^{6} \mathrm{~cm}^{-1}$.

The diagonal elements give rise to thermalization within the subbands; these processes do not change the number of particles within the subband.

In our example the nondiagonal matrix elements were divided into the three groups shown in Fig. 14 for the static screened Coulomb potential. The upper dashed curve shows $V\left(h_{m}, h_{n} ; h_{m}, h_{n}\right)$ or $V\left(h_{m}, h_{m} ; h_{n}, h_{n}\right)$, the solid line is $V\left(h_{m}, e_{i} ; e_{j}, h_{n}\right)$ or $V\left(e_{i}, h_{n} ; h_{m}, e_{j}\right)$, and the lower dashed line shows $V\left(e_{j}, e_{i} ; e_{j}, e_{i}\right)$ or $V\left(e_{i}, e_{i} ; e_{j}, e_{j}\right)$. In all cases both $i \neq j$ and $m \neq n$. The direct or exchange nondiagonal elements are responsible to a net exchange of carriers between the subbands, hence causing the occupation of the subbands to change. The diagonal elements of Fig. 13 are seen to be about an order of magnitude larger than the nondiagonal elements of Fig. 14.

\section{APPENDIX B: DERIVATION OF THE SBE'S}

The dynamics of the expectation values of the operator $\mathcal{O}$ may reside in the density operator $\rho$ or in the operator $\mathcal{O}$ itself, depending on whether we work in the Schrödinger or the Heisenberg picture, respectively. In this work the Heisenberg picture is used, and the dynamic equations are given by

$$
\dot{\mathcal{O}}=\frac{i}{\hbar}[H, \mathcal{O}]
$$

Taking the system Hamiltonian as the sum of Eqs. (3), (4), and (5), using fermion anticommutation relations, and some index manipulations leads to the rate equations for the expectation values $n_{e_{i}, \mathbf{p}}, n_{h_{i}, \mathbf{p}}$ for the electron and hole numbers and for the dipole expectation value $p_{\mathbf{p}}$ :

$$
\begin{aligned}
& \frac{d}{d t} n_{e_{i}, \mathbf{p}}=-\frac{1}{i \hbar}\left\langle\left[H, a_{e_{i}, \mathbf{p}}^{\dagger} a_{e_{i}, \mathbf{p}}\right]\right\rangle=\frac{1}{i \hbar}\left[-\mathbf{E}(t) \cdot\left(\boldsymbol{\mu}_{\mathbf{p}} p_{\mathbf{p}}^{*}-\boldsymbol{\mu}_{\mathbf{p}}^{*} p_{\mathbf{p}}\right)\right] \delta_{e_{i}, e_{1}}+\frac{1}{i \hbar} \sum_{e_{m}, \mathbf{k}^{\prime}, \mathbf{q}}\left[\sum_{h_{j}^{\prime}, h_{j}} V_{q}\left(e_{m}, h_{j}^{\prime} ; h_{j}, e_{i}\right)\right. \\
& \times\left\langle a_{e_{m}, \mathbf{p}+\mathbf{q}}^{\dagger} a_{e_{i}, \mathbf{p}} b_{h_{j}^{\prime},-\mathbf{k}^{\prime}-\mathbf{q}}^{\dagger} b_{h_{j},-\mathbf{k}^{\prime}}\right\rangle-V_{q}\left(e_{i}, h_{j}^{\prime} ; h_{j}, e_{m}\right)\left\langle a_{e_{i}, \mathbf{p}}^{\dagger} a_{e_{m}, \mathbf{p}+\mathbf{q}} b_{h_{j}^{\prime},-\mathbf{k}^{\prime}-\mathbf{q}}^{\dagger} b_{h_{j},-\mathbf{k}^{\prime}}\right\rangle \\
& \left.+\sum_{e_{j}^{\prime}, e_{j}} V_{q}\left(e_{j}^{\prime}, e_{i} ; e_{m}, e_{j}\right)\left\langle a_{e_{i}, \mathbf{p}^{\dagger}}^{\dagger} a_{e_{j}^{\prime}, \mathbf{k}^{\prime}-q}^{\dagger} a_{e_{j}, \mathbf{k}^{\prime}} a_{e_{m}, \mathbf{p}-\mathbf{q}}\right\rangle-V_{q}\left(e_{j}^{\prime}, e_{m} ; e_{i}, e_{j}\right)\left\langle a_{e_{m}, \mathbf{p}+\mathbf{q}}^{\dagger} a_{e_{j}^{\prime}, \mathbf{k}^{\prime}-q}^{\dagger} a_{e_{j}, \mathbf{k}^{\prime}} a_{e_{i}, \mathbf{p}}\right\rangle\right], \\
& \frac{d}{d t} n_{h_{i}, \mathbf{p}}=-\frac{1}{i \hbar}\left\langle\left[H, b_{h_{i},-\mathbf{p}}^{\dagger} b_{h_{i},-\mathbf{p}}\right]\right\rangle=\frac{1}{i \hbar}\left[-\mathbf{E}(t) \cdot\left(\boldsymbol{\mu}_{\mathbf{p}} p_{\mathbf{p}}^{*}-\boldsymbol{\mu}_{\mathbf{p}}^{*} p_{\mathbf{p}}\right)\right] \delta_{h_{i}, h_{1}} \\
& +\frac{1}{i \hbar} \sum_{h_{m}, \mathbf{k}^{\prime}, \mathbf{q}}\left[\sum_{h_{j}^{\prime}, h_{j}} V_{q}\left(h_{j}^{\prime}, h_{i} ; h_{m}, h_{j}\right)\left\langle b_{h_{i},-\mathbf{p}}^{\dagger} b_{h_{j}^{\prime},-\mathbf{k}^{\prime}+\mathbf{q}}^{\dagger} b_{h_{j},-\mathbf{k}^{\prime}} b_{h_{m},-\mathbf{p}-\mathbf{q}}\right\rangle-V_{q}\left(h_{j}^{\prime}, h_{m} ; h_{i}, h_{j}\right)\right. \\
& \times\left\langle b_{h_{m},-\mathbf{p}-\mathbf{q}}^{\dagger} b_{h_{j}^{\prime},-\mathbf{k}^{\prime}+\mathbf{q}}^{\dagger} b_{h_{j},-\mathbf{k}^{\prime}} b_{h_{i},-\mathbf{p}}\right\rangle+\sum_{e_{j}^{\prime}, e_{j}} V_{q}\left(h_{m}, e_{j}^{\prime} ; e_{j}, h_{i}\right)\left\langle b_{h_{m},-\mathbf{p}-\mathbf{q}}^{\dagger} b_{h_{i},-\mathbf{p}} a_{e_{j}^{\prime}, \mathbf{k}^{\prime}+\mathbf{q}}^{\dagger} a_{e_{j}, \mathbf{k}^{\prime}}\right\rangle \\
& \left.-V_{q}\left(h_{i}, e_{j}^{\prime} ; e_{j}, h_{m}\right)\left\langle b_{h_{i},-\mathbf{p}}^{\dagger} b_{h_{m},-\mathbf{p}-\mathbf{q}} a_{e_{j}^{\prime}, \mathbf{k}^{\prime}-\mathbf{q}}^{\dagger} a_{e_{j}, \mathbf{k}^{\prime}}\right\rangle\right],
\end{aligned}
$$




$$
\begin{aligned}
\frac{d}{d t} p_{\mathbf{p}}= & -\frac{1}{i \hbar}\left\langle\left[H, b_{h_{1},-\mathbf{p}} a_{e_{1}, \mathbf{p}}\right]\right\rangle=\frac{1}{i \hbar}\left[\left(\varepsilon_{e_{1}, \mathbf{p}}+\varepsilon_{h_{1}, \mathbf{p}}\right) p_{\mathbf{p}}+\mathbf{E}(t) \cdot \mu_{\mathbf{p}} g\left(n_{h_{1}, \mathbf{p}}+n_{e_{1}, \mathbf{p}}-1\right)+\sum_{\mathbf{q}} V_{q}\left(e_{1}, h_{1} ; h_{1}, e_{1}\right)\left\langle p_{e_{1}, h_{1}, \mathbf{p}-\mathbf{q}}\right\rangle\right] \\
& +\frac{1}{i \hbar} \sum_{\mathbf{k}^{\prime}, \mathbf{q}}\left[\sum_{h_{j}^{\prime}, h_{j}, e_{m}} V_{q}\left(e_{1}, h_{j}^{\prime} ; h_{j}, e_{m}\right)\left\langle b_{h_{j}^{\prime},-\mathbf{k}^{\prime}+\mathbf{q}^{\prime}} b_{h_{j},-\mathbf{k}^{\prime}} a_{e_{m}, \mathbf{p}+\mathbf{q}} b_{h_{1},-\mathbf{p}}\right\rangle+\sum_{e_{j}^{\prime}, h_{j}, e_{m}} V_{q}\left(e_{j}^{\prime}, h_{1} ; h_{j}, e_{m}\right)\right. \\
& \times\left\langle a_{e_{j}^{\prime}, \mathbf{k}^{\prime}+q}^{\dagger} b_{h_{j},-\mathbf{p}+\mathbf{q}} a_{e_{m}, \mathbf{k}^{\prime}} a_{e_{1}, \mathbf{p}}\right\rangle-\sum_{h_{j}^{\prime}, h_{j}, h_{m}} V_{q}\left(h_{j}^{\prime}, h_{1} ; h_{j}, h_{m}\right)\left\langle b_{h_{j}^{\prime},-\mathbf{k}^{\prime}-\mathbf{q}}^{\dagger} b_{h_{j},-\mathbf{p}-\mathbf{q}} b_{h_{m},-\mathbf{k}^{\prime}} a_{e_{1}, \mathbf{p}}\right\rangle \\
& \left.-\sum_{e_{j}^{\prime}, e_{j}, e_{m}} V_{q}\left(e_{j}^{\prime}, e_{1} ; e_{j}, e_{m}\right)\left\langle a_{e_{j}^{\prime}, \mathbf{k}^{\prime}+q}^{\dagger} a_{e_{j}, \mathbf{p}+\mathbf{q}} a_{e_{m}, \mathbf{k}^{\prime}} b_{h_{1},-\mathbf{p}}\right\rangle\right] .
\end{aligned}
$$

The three expectation values $n_{e_{i}, \mathbf{p}}, n_{h_{i}, \mathbf{p}}$, and $p_{\mathbf{p}}$ are twooperator expectation values, and their rate equations are given in terms of four-operator expectation values. By using Eq. (B1) on the four-operator expectation values we get rate equations given in terms of six-operator expectation values. Continuing the iteration gives an infinite hierarchy of coupled equations, known as the BBGYK hierarchy. The set of equations may be truncated by nullifying the correlated part of the $2(N+1)$-operator expectation value in the rate equations for the $2 \mathrm{~N}$-operator expectation values. For the two-operator rate equations, this yields the SBE in the freecarrier model. For the four-operator expectation values it gives the SBE's in the interacting carrier model along with the collision equations. Each expectation value of a $2 \mathrm{~N}$ operator can be decomposed into all possible products of expectation values of lower-order operators plus a contribution from the correlated part of the $2 N$ operator which cannot be expressed as product of lower-order terms (this is the wellknown Wick theorem ${ }^{4,29}$ ).

Since we are interested in the spectral hole burning effect, it is necessary to evaluate the four-operator correlations. For this case the six-operator correlations are neglected. It can be done by using the Hartree-Fock factorization method, in which the first-order contribution of the expectation value of a $2 \mathrm{~N}$-operator expectation value is given by all possible expectation value products of two-operator expectation values. For example, the third four-operator expectation value in Eq. (B2) yields

$$
\begin{aligned}
& \left\langle a_{e_{i}, \mathbf{p}}^{\dagger} a_{e_{j}^{\prime}, \mathbf{k}^{\prime}-q}^{\dagger} a_{e_{j}, \mathbf{k}^{\prime}} a_{e_{m}, \mathbf{p}-\mathbf{q}}\right\rangle \\
& =-n_{e_{i}, \mathbf{p}^{\prime}} n_{e_{m}, \mathbf{p}-\mathbf{q}} \delta_{\mathbf{p}, \mathbf{k}^{\prime}} \delta_{e_{i}, e_{j}} \delta_{e_{j}^{\prime}, e_{m}} \\
& \quad+\delta\left\langle a_{e_{i}, \mathbf{p}^{\prime}}^{\dagger} a_{e_{j}^{\prime}, \mathbf{k}^{\prime}-q}^{\dagger} a_{e_{j}, \mathbf{k}^{\prime}} a_{e_{m}, \mathbf{p}-\mathbf{q}}\right\rangle,
\end{aligned}
$$

where the first term is the Hartree-Fock factorization process contribution and the last $\delta$ term is the four-operator correlation (neglecting $\delta_{\mathbf{q}, 0}$ terms due to charge neutrality). Repeating this procedure for all of the four-operator expectation values in Eqs. (B2)-(B4) and after some algebra and index manipulations, we get the set of equations given by Eqs. (6)-(10).

In order to write explicit terms for the collision parts of the SBE's (11) and (12), we need to write the equations of the four-operator correlation term [the last $\delta$ term in Eq. (B5)], for each correlation in Eqs. (B2)-(B4). In the next step, keeping only the Hartree-Fock factored terms and replacing the bare Coulomb potential with the screened potential we get the Eqs. (11) and (12) . Following this procedure, we find the asymptotic limit $t \rightarrow \infty$ of the quantum kinetic equation for the four-operator correlation equation, in which we assume that the relaxation time of the correlation part is short compared with that of the single-particle (Bogoliubov approximation ${ }^{6,30}$ ). Using this procedure, we get relaxed correlation functions determined in terms of nonequilibrium two-operator expectation values, which yields a scattering integral along with the screened potential.

\section{APPENDIX C: COLLISION RATES}

In the following scattering rate terms, if $\alpha$ denotes the conduction band, then $\beta$ denotes the valence band and vice versa. One can get the outscattering rate terms $X_{\lambda, \mathbf{p}}^{\text {out }}$ by replacing $n_{\lambda, \mathbf{p}} \leftrightarrow 1-n_{\lambda, \mathbf{p}}$ in Eqs. (C3), (C4), and (C6). Before writing the explicit form of the rate terms in Eqs. (11) and (12), we introduce the following two Coulomb matrix elements:

$$
\begin{aligned}
& \widetilde{V}_{\mathbf{k}, \mathbf{p}, \mathbf{q}}^{1}\left(\lambda_{j}, \lambda_{m} ; \lambda_{n}, \lambda_{i}\right) \\
& \quad=V_{s, q}\left(\lambda_{j}, \lambda_{m} ; \lambda_{n}, \lambda_{i}\right)-V_{s,|\mathbf{k}-\mathbf{p}-\mathbf{q}|}\left(\lambda_{j}, \lambda_{m} ; \lambda_{i}, \lambda_{n}\right),
\end{aligned}
$$

$$
\begin{aligned}
& \widetilde{V}_{\mathbf{k}, \mathbf{p}, \mathbf{q}}^{2}\left(\lambda_{j}, \lambda_{m} ; \lambda_{n}, \lambda_{i}\right) \\
& \quad=V_{s, q}^{*}\left(\lambda_{j}, \lambda_{m} ; \lambda_{n}, \lambda_{i}\right) \cdot \widetilde{V}_{\mathbf{k}, \mathbf{p}, \mathbf{q}}^{1}\left(\lambda_{j}, \lambda_{m} ; \lambda_{n}, \lambda_{i}\right),
\end{aligned}
$$




$$
\begin{aligned}
& \Gamma_{\alpha_{i}, \mathbf{p}}^{i n}=\frac{2 \pi}{\hbar} \sum_{\mathbf{k}, \mathbf{q}, \alpha_{j}}\left[\sum_{\alpha_{n}, \alpha_{m}} \delta\left(\varepsilon_{\alpha_{j}, \mathbf{p}+\mathbf{q}}+\varepsilon_{\alpha_{m}, \mathbf{k}-\mathbf{q}}-\varepsilon_{\alpha_{n}, \mathbf{k}}-\varepsilon_{\alpha_{i}, \mathbf{p}}\right) \cdot\left[\widetilde{V}_{\mathbf{k}, \mathbf{p}, \mathbf{q}}^{2}\left(\alpha_{j}, \alpha_{m} ; \alpha_{n}, \alpha_{i}\right)\right] \cdot\left[\left(1-n_{\alpha_{n}, \mathbf{k}}\right) \cdot n_{\alpha_{m}, \mathbf{k}-\mathbf{q}} \cdot n_{\alpha_{j}, \mathbf{p}+\mathbf{q}}\right]\right. \\
& \left.+\sum_{\beta_{n}, \beta_{m}} \delta\left(\varepsilon_{\alpha_{j}, \mathbf{p}+\mathbf{q}}-\varepsilon_{\beta_{n}, \mathbf{k}-\mathbf{q}}+\varepsilon_{\beta_{m}, \mathbf{k}}-\varepsilon_{\alpha_{i}, \mathbf{p}}\right)\left[\left|V_{s, q}\left(\alpha_{j}, \beta_{m} ; \beta_{n}, \alpha_{i}\right)\right|^{2} \cdot\left(1-n_{\beta_{n}, \mathbf{k}-\mathbf{q}}\right) \cdot n_{\beta_{m}, \mathbf{k}} \cdot n_{\alpha_{j}, \mathbf{p}+\mathbf{q}}\right]\right], \\
& \Delta_{\alpha_{i}, \mathbf{p}}^{i n,(2)}=\frac{-\pi}{\hbar} \sum_{\mathbf{k}, \mathbf{q}, \alpha_{j}}\left\{\left[\delta\left(\varepsilon_{\alpha_{j}, \mathbf{p}+\mathbf{q}}+\varepsilon_{\alpha_{1}, \mathbf{k}-\mathbf{q}}-\varepsilon_{\alpha_{1}, \mathbf{k}}-\varepsilon_{\alpha_{i}, \mathbf{p}}\right) \cdot n_{\alpha_{j}, \mathbf{p}+\mathbf{q}}+\delta\left(\varepsilon_{\alpha_{j}, \mathbf{p}+\mathbf{q}}-\varepsilon_{\beta_{1}, \mathbf{k}-\mathbf{q}}+\varepsilon_{\beta_{1}, \mathbf{k}}\right.\right.\right. \\
& \left.\left.\left.-\varepsilon_{\alpha_{i}, \mathbf{p}}\right) \cdot n_{\alpha_{j}, \mathbf{p}+\mathbf{q}}\right] \cdot\left[V_{s, q}^{*}\left(\alpha_{j}, \beta_{1} ; \beta_{1}, \alpha_{i}\right) \cdot \widetilde{V}_{\mathbf{k}, \mathbf{p}, \mathbf{q}}^{1}\left(\alpha_{j}, \alpha_{1} ; \alpha_{1}, \alpha_{i}\right) \cdot p_{\mathbf{k}-\mathbf{q}}^{*} \cdot p_{\mathbf{k}}+\text { c.c. }\right]\right\}-\frac{\pi}{\hbar} \sum_{\mathbf{k}, \mathbf{q}, \beta_{j}}\left\{\delta \left(\varepsilon_{\alpha_{j}, \mathbf{p}+\mathbf{q}}-\varepsilon_{\beta_{1}, \mathbf{k}-\mathbf{q}}\right.\right. \\
& \left.\left.+\varepsilon_{\beta_{j}, \mathbf{k}}-\varepsilon_{\alpha_{i}, \mathbf{p}}\right) \cdot n_{\beta_{j}, \mathbf{k}} \cdot\left[V_{s, q}^{*}\left(\alpha_{1}, \beta_{j} ; \beta_{1}, \alpha_{i}\right) \cdot V_{s,|\mathbf{k}-\mathbf{p}-\mathbf{q}|}\left(\alpha_{1}, \beta_{j} ; \beta_{1}, \alpha_{i}\right) \cdot p_{\mathbf{k}-\mathbf{q}}^{*} \cdot p_{\mathbf{p}+\mathbf{q}}+\text { c.c. }\right]\right\}, \\
& \Delta_{\alpha, \mathbf{p}}^{(1)}=\frac{\pi}{\hbar} \sum_{\mathbf{k}, \mathbf{q}}\left[\sum _ { \alpha _ { m } , \alpha _ { n } } \left[\delta ( \varepsilon _ { \alpha _ { 1 } , \mathbf { p } + \mathbf { q } } + \varepsilon _ { \alpha _ { m } , \mathbf { k } - \mathbf { q } } - \varepsilon _ { \alpha _ { n } , \mathbf { k } } - \varepsilon _ { \alpha _ { 1 } , \mathbf { p } } ) \cdot \left(n_{\alpha_{n}, \mathbf{k}}\right.\right.\right. \\
& \left.\left.-n_{\alpha_{m}, \mathbf{k}-\mathbf{q}}\right)\right] \cdot\left[V_{s, q}^{*}\left(\alpha_{m}, \beta_{1} ; \beta_{1}, \alpha_{n}\right) \cdot \widetilde{V}_{\mathbf{k}, \mathbf{p}, \mathbf{q}}^{1}\left(\alpha_{m}, \alpha_{1} ; \alpha_{1}, \alpha_{n}\right) \cdot p_{\mathbf{p}+\mathbf{q}}^{*}\right]+\frac{\pi}{\hbar} \sum_{\mathbf{k}, \mathbf{q}}\left[\sum _ { \beta _ { m } , \beta _ { n } } \left[\delta \left(\varepsilon_{\alpha_{1}, \mathbf{p}+\mathbf{q}}-\varepsilon_{\beta_{m}, \mathbf{k}-\mathbf{q}}+\varepsilon_{\beta_{n}, \mathbf{k}}\right.\right.\right. \\
& \left.\left.-\varepsilon_{\alpha_{1}, \mathbf{p}}\right) \cdot\left(n_{\beta_{m}, \mathbf{k}-\mathbf{q}}-n_{\beta_{n}, \mathbf{k}}\right)\right] \cdot\left[V_{s, q}^{*}\left(\beta_{m}, \alpha_{1} ; \alpha_{1}, \beta_{n}\right) \cdot \widetilde{V}_{\mathbf{k}, \mathbf{p}, \mathbf{q}}^{1}\left(\beta_{m}, \beta_{1} ; \beta_{1}, \beta_{n}\right) \cdot p_{\mathbf{p}+\mathbf{q}}^{*}\right]+\frac{\pi}{\hbar} \sum_{\mathbf{k}, \mathbf{q}}\left[\sum _ { \beta _ { m } , \alpha _ { n } } \left[\delta \left(\varepsilon_{\alpha_{n}, \mathbf{p}+\mathbf{q}}\right.\right.\right. \\
& \left.\left.\left.-\varepsilon_{\beta_{m}, \mathbf{k}-\mathbf{q}}+\varepsilon_{\beta_{1}, \mathbf{k}}-\varepsilon_{\alpha_{1}, \mathbf{p}}\right) \cdot\left(n_{\beta_{m}, \mathbf{k}-\mathbf{q}}-n_{\alpha_{n}, \mathbf{p}+\mathbf{q}}\right)\right] \cdot\left[V_{s, q}^{*}\left(\alpha_{1}, \beta_{m} ; \beta_{1}, \alpha_{n}\right) \cdot V_{s,|\mathbf{k}-\mathbf{p}-\mathbf{q}|}\left(\alpha_{1}, \beta_{m} ; \beta_{1}, \alpha_{n}\right) \cdot p_{\mathbf{k}}^{*}\right]\right], \\
& \Lambda_{\alpha_{1}, \mathbf{p}}^{i n,(1)}=\frac{\pi}{\hbar} \sum_{\mathbf{k}, \mathbf{q}}\left[\sum _ { \alpha _ { m } , \alpha _ { n } } \left[\delta\left(\varepsilon_{\beta_{1}, \mathbf{p}+\mathbf{q}}+\varepsilon_{\alpha_{m}, \mathbf{k}}-\varepsilon_{\alpha_{n}, \mathbf{k}-\mathbf{q}}-\varepsilon_{\beta_{1}, \mathbf{p}}\right) \cdot(1\right.\right. \\
& \left.\left.-n_{\alpha_{m}, \mathbf{k}}\right)\right] \cdot n_{\alpha_{n}, \mathbf{k}-\mathbf{q}} \cdot\left[V_{s, q}^{*}\left(\alpha_{m}, \beta_{1} ; \beta_{1}, \alpha_{n}\right) \cdot \widetilde{V}_{\mathbf{k}, \mathbf{p}, \mathbf{q}}^{1}\left(\alpha_{m}, \alpha_{1} ; \alpha_{1}, \alpha_{n}\right) \cdot p_{\mathbf{p}+\mathbf{q}}\right]+\frac{\pi}{\hbar} \sum_{\mathbf{k}, \mathbf{q}}\left[\sum _ { \beta _ { m } , \beta _ { n } } \left[\delta \left(\varepsilon_{\beta_{1}, \mathbf{p}+\mathbf{q}}+\varepsilon_{\beta_{m}, \mathbf{k}-\mathbf{q}}\right.\right.\right. \\
& \left.\left.\left.-\varepsilon_{\beta_{n}, \mathbf{k}}-\varepsilon_{\beta_{1}, \mathbf{p}}\right) \cdot\left(1-n_{\beta_{m}, \mathbf{k}-\mathbf{q}}\right)\right] \cdot n_{\beta_{n}, \mathbf{k}} \cdot\left[V_{s, q}^{*}\left(\beta_{n}, \alpha_{1} ; \alpha_{1}, \beta_{m}\right) \cdot \widetilde{V}_{\mathbf{k}, \mathbf{p}, \mathbf{q}}^{1}\left(\beta_{n}, \beta_{1} ; \beta_{1}, \beta_{m}\right) \cdot p_{\mathbf{p}+\mathbf{q}}\right]\right] \\
& +\frac{\pi}{\hbar} \sum_{\mathbf{k}, \mathbf{q}}\left[\sum_{\beta_{m}, \alpha_{n}}\left[\delta\left(\varepsilon_{\beta_{m}, \mathbf{p}+\mathbf{q}}-\varepsilon_{\alpha_{1}, \mathbf{k}}+\varepsilon_{\alpha_{n}, \mathbf{k}-\mathbf{q}}-\varepsilon_{\beta_{1}, \mathbf{p}}\right) \cdot n_{\alpha_{n}, \mathbf{k}-\mathbf{q}}\right] \cdot(1\right. \\
& \left.\left.-n_{\beta_{m}, \mathbf{p}+\mathbf{q}}\right) \cdot\left[V_{s, q}^{*}\left(\beta_{m}, \alpha_{1} ; \alpha_{n}, \beta_{1}\right) \cdot V_{s,|\mathbf{k}-\mathbf{p}-\mathbf{q}|}\left(\beta_{m}, \alpha_{1} ; \alpha_{n}, \beta_{1}\right) \cdot p_{\mathbf{k}}\right]\right] \text {, } \\
& \Lambda_{\alpha_{1}, \mathbf{p}}^{(2)}=\frac{\pi}{\hbar} \sum_{\mathbf{k}, \mathbf{q}, \alpha_{m}}\left\{\delta\left(\varepsilon_{\alpha_{m}, \mathbf{p}+\mathbf{q}}-\varepsilon_{\beta_{1}, \mathbf{k}-\mathbf{q}}+\varepsilon_{\beta_{1}, \mathbf{k}}-\varepsilon_{\alpha_{1}, \mathbf{p}}\right) \cdot\left[V_{s, q}^{*}\left(\alpha_{m}, \beta_{1} ; \beta_{1}, \alpha_{1}\right) \cdot \widetilde{V}_{\mathbf{k}, \mathbf{p}, \mathbf{q}}^{1}\left(\alpha_{m}, \alpha_{1} ; \alpha_{1}, \alpha_{1}\right) \cdot p_{\mathbf{k}-\mathbf{q}}^{*} p_{\mathbf{k}}\right]\right\} \\
& +\left\{\delta\left(\varepsilon_{\alpha_{m}, \mathbf{p}+\mathbf{q}}+\varepsilon_{\alpha_{1}, \mathbf{k}-\mathbf{q}}-\varepsilon_{\alpha_{1}, \mathbf{k}}-\varepsilon_{\alpha_{1}, \mathbf{p}}\right) \cdot\left[V_{s, q}^{*}\left(\alpha_{1}, \beta_{1} ; \beta_{1}, \alpha_{m}\right) \cdot \widetilde{V}_{\mathbf{k}, \mathbf{p}, \mathbf{q}}^{1}\left(\alpha_{1}, \alpha_{1} ; \alpha_{1}, \alpha_{m}\right) \cdot p_{\mathbf{k}-\mathbf{q}} p_{\mathbf{k}}^{*}\right]\right\} \\
& +\left\{\delta\left(\varepsilon_{\beta_{1}, \mathbf{p}+\mathbf{q}}+\varepsilon_{\alpha_{m}, \mathbf{k}}-\varepsilon_{\alpha_{1}, \mathbf{k}-\mathbf{q}}-\varepsilon_{\beta_{1}, \mathbf{p}}\right) \cdot\left[V_{s, q}^{*}\left(\alpha_{m}, \beta_{1} ; \beta_{1}, \alpha_{1}\right) \cdot V_{|\mathbf{k}-\mathbf{p}-\mathbf{q}|}\left(\alpha_{m}, \alpha_{1} ; \alpha_{1}, \alpha_{1}\right) \cdot p_{\mathbf{k}-\mathbf{q}}^{*} p_{\mathbf{p}+\mathbf{q}}\right]\right\}, \\
& \Lambda_{\alpha_{1}, \mathbf{p}}^{(3)}=\frac{-\pi}{\hbar} \sum_{\mathbf{k}, \mathbf{q}}\left\{\delta\left(\varepsilon_{\alpha_{1}, \mathbf{p}+\mathbf{q}}+\varepsilon_{\alpha_{1}, \mathbf{k}-\mathbf{q}}-\varepsilon_{\alpha_{1}, \mathbf{k}}-\varepsilon_{\alpha_{1}, \mathbf{p}}\right) \cdot\left[V_{s, q}^{*}\left(\alpha_{1}, \alpha_{1} ; \alpha_{1}, \alpha_{1}\right) \cdot \widetilde{V}_{\mathbf{k}, \mathbf{p}, \mathbf{q}}^{1}\left(\beta_{1}, \beta_{1} ; \beta_{1}, \beta_{1}\right) \cdot p_{\mathbf{k}-\mathbf{q}} p_{\mathbf{k}}^{*} p_{\mathbf{p}+\mathbf{q}}\right]\right\} \\
& \times \frac{-\pi}{\hbar} \sum_{\mathbf{k}, \mathbf{q}}\left\{\delta\left(\varepsilon_{\beta_{1}, \mathbf{p}+\mathbf{q}}-\varepsilon_{\alpha_{1}, \mathbf{k}-\mathbf{q}}+\varepsilon_{\alpha_{1}, \mathbf{k}}-\varepsilon_{\beta_{1}, \mathbf{p}}\right) \cdot\left[\left|V_{s, q}^{*}\left(\alpha_{1}, \beta_{1} ; \beta_{1}, \alpha_{1}\right)\right|^{2} \cdot p_{\mathbf{k}-\mathbf{q}}^{*} p_{\mathbf{k}} p_{\mathbf{p}+\mathbf{q}}\right]\right\} .
\end{aligned}
$$


Each of these collision integral expressions $(\Gamma, \Delta, \Lambda)$ denotes a rate which is computed via a discrete summation over subbands and three integrations. For each $p$ the integration is held over $q$, over the angle between $\mathbf{p}$ and $\mathbf{q}(\varphi)$, and over $k$. In the Markovian approximation, the angle between $\mathbf{k}$ and $\mathbf{q}$ $(\phi)$ is determined by the energy Dirac $\delta$ function. There are, however, subtle cases where the integrand diverges, such as longitudinal scattering at diagonal elements $(\mathbf{k} \cdot \mathbf{q}= \pm k q)$; these divergences can be removed by variable manipulations, which change the limits of the $k$ integration. These limits are different for diagonal scattering and nondiagonal scattering due to the different subband mass considered in our example. There are two possible $\phi$ angles for each $k\left(\phi_{k}\right.$ and $2 \pi$ $-\phi_{k}$ ). The direct terms are the same for both angles because their scalar product magnitudes are equal. These terms contain products of the form $V_{s, q}(\cdots) \cdot V_{s, q}^{*}(\cdots)$. On the other hand, in the exchange terms which contain products of the form $V_{s, q}^{*}(\cdots) \cdot V_{s,|\mathbf{k}-\mathbf{p}-\mathbf{q}|}(\cdots)$, the magnitude of the vector $|\mathbf{k}-\mathbf{p}-\mathbf{q}|$ is affected differently by $\phi_{k}$ and $2 \pi-\phi_{k}$ due to the angle between $\mathbf{k}$ and $\mathbf{p}(\varphi+\phi$ and $\varphi-\phi)$. All in all the direct terms have a factor of 2 whereas the exchange terms split into two cases. It can be seen that the $\varphi$ integration from 0 to $\pi$ and from $\pi$ to $2 \pi$ yields the same results for the direct and exchange cases and, hence, one can integrate only over the first region and have an additional overall factor of 2 .
*Electronic address: dery@techunix.technion.ac.il

${ }^{1}$ L. P. Kadanoff and G. Baym, Quantum Statistical Mechanics (Benjamin, New York, 1962).

${ }^{2}$ L. V. Keldish, Sov. Phys. JETP 20, 1018 (1963).

${ }^{3} \mathrm{H}$. Haug, in Optical Nonlinearities and Instabilities in Semiconductors, edited by H. Haug (Academic, San Diego, 1988), p. 53.

${ }^{4}$ G. D. Mahan, Many Particle Physics, 2nd ed. (Plenum, New York, 1993).

${ }^{5}$ M. Lindberg and S. W. Koch, Phys. Rev. B 38, 3342 (1988).

${ }^{6}$ H. W. Wyld and B. D. Fried, Ann. Phys. (N.Y.) 23, 374 (1963).

${ }^{7}$ T. Kuhn and F. Rossi, Phys. Rev. B 46, 7496 (1992).

${ }^{8}$ T. Kuhn, in Theory of Transport Properties of Semiconductor NanoStructures, edited by E. Schöll (Chapman \& Hall, London, 1998), pp. 173-214.

${ }^{9}$ E. D. Siggia and P. C. Kwok, Phys. Rev. B 2, 1024 (1970).

${ }^{10}$ S. M. Goodnick and P. Lugli, Phys. Rev. B 38, 10135 (1988).

${ }^{11}$ R. Binder, D. Scott, A. E. Paul, M. Lindberg, K. Henneberger, and S. W. Koch, Phys. Rev. B 45, 1107 (1992).

${ }^{12}$ S. M. Goodnick and P. Lugli, Phys. Rev. B 37, 2578 (1988).

${ }^{13}$ F. Prengel and E. Schöll, Phys. Rev. B 59, 5806 (1999).

${ }^{14}$ H. C. Schneider, F. Jahnke, and S. W. Koch, Quantum Semiclassic. Opt. 9, 693 (1997).

${ }^{15}$ W. W. Chow, S. W. Koch, and M. Sargent III, SemiconductorLaser Physics (Springer, Berlin, 1994).

${ }^{16}$ S. Hughes, Phys. Rev. A 58, 2567 (1998).

${ }^{17}$ K. El Sayed, L. Bányai, and H. Haug, Phys. Rev. B 50, 1541 (1994).
${ }^{18}$ F. X. Camescasse, A. Alexandrou, D. Hulin, L. Bányai, D. B. Tran Thoai, and H. Haug, Phys. Rev. Lett. 77, 5429 (1996).

${ }^{19}$ Q. T. Vu, L. Bányai, H. Haug, F. X. Camescasse, J. P. Likforman, and A. Alexandrou, Phys. Rev. B 59, 2760 (1999).

${ }^{20}$ B. Mieck, H. Haug, W. A. Hügel, M. F. Heinrich, and M. Wegener, Phys. Rev. B 62, 2686 (2000).

${ }^{21}$ Silvan S. Schweber, An Introduction to Relativistic Quantum Field Theory (Row, Peterson, Evanston, 1961).

${ }^{22}$ D. Gershoni, C. H. Henry, and G. A. Baraff, IEEE J. Quantum Electron. QE-29, 2433 (1993).

${ }^{23}$ H. Dery, B. Tromborg, and G. Eisenstein (unpublished).

${ }^{24}$ See, for example, B. K. Ridley, in Hot Carrier in Semiconductor NanoStructures, edited by J. Shah (Academic, San Diego, 1992), pp. $17-50$.

${ }^{25}$ K. Huang and B. Zhu, Phys. Rev. B 38, 13377 (1988).

${ }^{26}$ M. P. Chamberlain, M. Babiker, Solid-State Electron. 32, 1675 (1989).

${ }^{27}$ V. M. Menon, W. D. Goodhue, A. S. Karakashian, and L. R. Ram-Mohan, J. Appl. Phys. 88, 5262 (2000).

${ }^{28}$ H. Haug and S. W. Koch, Quantum Theory of the Optical and Electronic Properties of Semiconductor (World Scientific, Singapore, 1990).

${ }^{29}$ D. J. Rowe, Nuclear Collective Motion, Models and Theory (Methuen, London, 1970).

${ }^{30}$ N. Bogoliubov, in Statistical Mechanics (North-Holland, Amsterdam, 1962), Vol. I. 